\title{
Half-metallicity and Slater-Pauling behavior in the ferromagnetic Heusler alloys
}

\author{
Iosif Galanakis and Peter H. Dederichs \\ Institut für Festkörperforschung, Forschungszentrum Jülich, D-52425 Jülich, \\ Germany I.Galanakis@fz-juelich.de and P.H.Dederichs@fz-juelich.de
}

\section{Introduction}

Half-metallic ferromagnets represent a new class of materials which attracted a lot of attention due to their possible applications in spintronics (also known as magnetoelectronics) [1. Adding the spin degree of freedom to the conventional electronic devices has several advantages like non-volatility, increased data processing speed, decreased electric power consumption and increased integration densities [2]. The current advances in new materials and especially in the half-metals are promising for engineering new spintronic devices in the near future [2. In these materials the two spin bands show a completely different behavior. While the majority spin band (referred also as spin-up band) shows the typical metallic behavior, the minority spin band (spin-down band) exhibits a semiconducting behavior with a gap at the Fermi level. Therefore such half-metals are ferromagnets and can be considered as hybrids between metals and semiconductors. A schematic representation of the density of states of a half-metal as compared to a normal metal and a normal semiconductor is shown in figure 1 . The spinpolarization at the Fermi level is $100 \%$ and therefore these compounds should have a fully spinpolarised current and might be able to yield a $100 \%$ spininjection and thus to maximize the efficiency of magnetoelectronic devices [3].
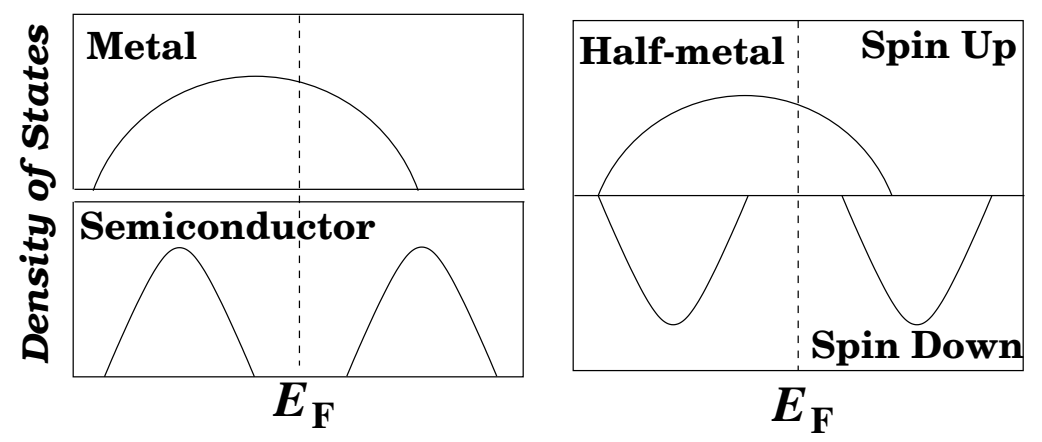

Fig. 1. Schematic representation of the density of states for a half-metal with respect to normal metals and semiconductors. 
Heusler alloys [4 have attracted during the last century a great interest due to the possibility to study in the same family of alloys a series of interesting diverse magnetic phenomena like itinerant and localized magnetism, antiferromagnetism, helimagnetism, Pauli paramagnetism or heavy-fermionic behavior [5, 6, 7, 8, The first Heusler alloys studied were crystallizing in the $L 2_{1}$ structure which consists of 4 fcc sublattices. Afterwards, it was discovered that it is possible to leave one of the four sublattices unoccupied $\left(C 1_{b}\right.$ structure). The latter compounds are often called half- or semi-Heusler alloys, while the $L 2_{1}$ compounds are referred to as full-Heusler alloys. In a pioneering theory paper in 1983 de Groot and his collaborators [9] showed by using first-principles electronic structure calculations that one of the half-Heusler compounds, NiMnSb, is a half-metal, i.e. the minority band is semiconducting with a gap at the Fermi level $E_{F}$, leading to $100 \%$ spin polarization at $E_{F}$ as shown in figure 1 Other known half-metallic materials except the halfand full-Heusler alloys 10, 11, 12, 13, are some oxides $\left(e . g \mathrm{CrO}_{2}\right.$ and $\left.\mathrm{Fe}_{3} \mathrm{O}_{4}\right)$ 14, the manganites (e.g $\mathrm{La}_{0.7} \mathrm{Sr}_{0.3} \mathrm{MnO}_{3}$ ) 14, the double perovskites (e.g. $\mathrm{Sr}_{2} \mathrm{FeReO}_{6}$ ) [15], the pyrites (e.g $\left.\mathrm{CoS}_{2}\right)$ [16], the transition metal chalcogenides $(e . g \mathrm{CrSe})$ and pnictides $(e . g \mathrm{CrAs})$ in the zinc-blende or wurtzite structures 17, 18, 19, 20, the europium chalcogenides (e.g EuS) 21] and the diluted magnetic semiconductors (e.g Mn impurities in $\mathrm{Si}$ or GaAs) 22, 23. Although thin films of $\mathrm{CrO}_{2}$ and $\mathrm{La}_{0.7} \mathrm{Sr}_{0.3} \mathrm{MnO}_{3}$ have been verified to present practically $100 \%$ spin-polarization at the Fermi level at low temperatures 14. 24, the Heusler alloys remain attractive for technical applications like spin-injection devices [25], spin-filters [26, tunnel junctions [27, or GMR devices 28 due to their relatively high Curie temperature compared to these compounds [5].

The half-metallic character of NiMnSb in single crystals seems to have been well-established experimentally. Infrared absorption 29] and spin-polarized positron-annihilation 30. gave a spin-polarization of $\sim 100 \%$ at the Fermi level. Recently it has also become possible to grow high quality films of Heusler alloys, and it is mainly NiMnSb that has attracted the attention 31, 32, 33. Unfortunately these films were found not to be half-metallic [14, 34, 35, 36, 37]; a maximum value of $58 \%$ for the spin-polarization of NiMnSb was obtained by Soulen et al. [14. These polarization values are consistent with a small perpendicular magnetoresistance measured for NiMnSb in a spin-valve structure [38, a superconducting tunnel junction [27] and a tunnel magnetoresistive junction [39. Ristoiu et al. showed that during the growth of the NiMnSb thin films, $\mathrm{Sb}$ and then $\mathrm{Mn}$ atoms segregate to the surface, which is far from being perfect, thus decreasing the obtained spin-polarization [40. But when they removed the excess of Sb by flash annealing, they managed to get a nearly stoichiometric ordered alloy surface being terminated by a MnSb layer, which presented a spin-polarization of about $67 \pm 9 \%$ at room temperature [40]. 
Several groups have verified the half-metallic character of bulk NiMnSb using first-principles calculations 41, 42. Larson et al. have shown that the actual structure of $\mathrm{NiMnSb}$ is the most stable with respect to an interchange of the atoms 43 and Orgassa et al. showed that a few percent of disorder induce states within the gap but do not destroy the half-metallicity 44. Recently, Galanakis has shown by first-principle calculations that NiMnSb surfaces do not present 100\% spin-polarization [45] but Wijs and de Groot proposed that at some interfaces it is possible to restore the half-metallic character of NiMnSb [46. These results were also confirmed by Debernardi et al. who studied the interface between NiMnSb and GaAs [47. Jenkins and King studied by a pseudopotential technique the MnSb terminated (001) surface of NiMnSb and showed that there are two surface states at the Fermi level, which are well localized at the surface layer [48 and they persist even when the MnSb surface is covered by a Sb overlayer [49].

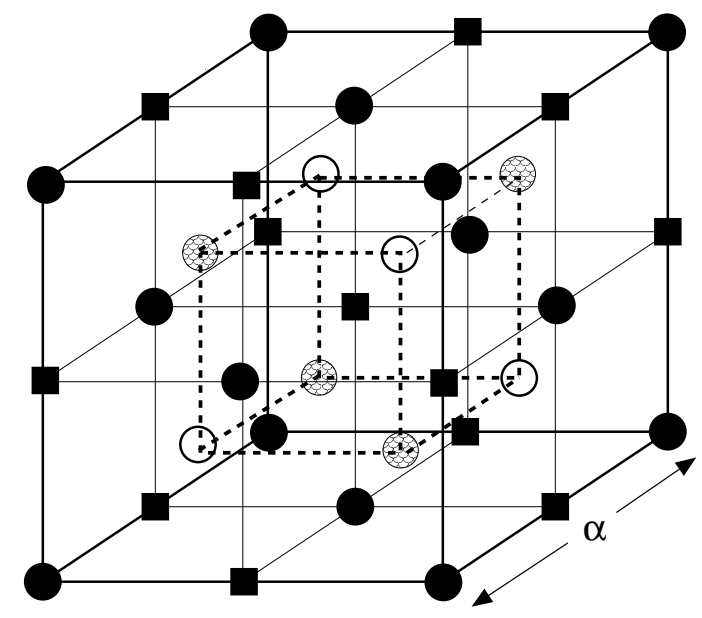

\section{Full-Heusler $\mathrm{X}_{2} \mathrm{YZ}\left[\mathrm{L2} \mathbf{O}_{1}\right]$ ○ $\boldsymbol{X} \bigcirc \boldsymbol{Y}$

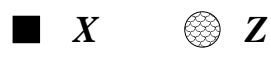

\section{Half-Heusler $X Y Z\left[C 1_{b}\right]$

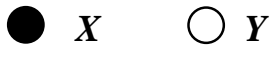 \\ - Void $Z$}

Fig. 2. $C 1_{b}$ and $L 2_{1}$ structures adapted by the half- and full-Heusler alloys. The lattice is consisted of 4 interprenatating f.c.c. lattice. The unit cell is that of a fcc lattice with four atoms as basis, e.g. CoMnSb: Co at (000), Mn at $\left(\frac{1}{4} \frac{1}{4} \frac{1}{4}\right)$, a vacant site at $\left(\frac{1}{2} \frac{1}{2} \frac{1}{2}\right)$ and $\mathrm{Sb}$ at $\left(\frac{3}{4} \frac{3}{4} \frac{3}{4}\right)$ in Wyckoff coordinates. In the case of the full Heusler alloys also the vacant site is occupied by a Co atom. Note also that if all atoms were identical, the lattice would be simply the bcc.

Webster and Ziebeck [50] and Suits [51] were the first to synthesize fullHeusler alloys containing $\mathrm{Co}$ and $\mathrm{Rh}$, respectively. Kübler et al. studied the mechanisms stabilizing the ferro- or the antiferromagnetism in these compounds [52. Ishida and collaborators have proposed that the compounds of the type $\mathrm{Co}_{2} \mathrm{MnZ}$, where $\mathrm{Z}$ stands for $\mathrm{Si}$ and $\mathrm{Ge}$, are half-metals 53 , 54]. Also the Heusler alloys of the type $\mathrm{Fe}_{2} \mathrm{MnZ}$ have been proposed to show halfmetallicity [55]. But Brown et al. [56] using polarized neutron diffraction mea- 
surements have shown that there is a finite very small spin-down density of states (DOS) at the Fermi level instead of an absolute gap in agreement with the ab-initio calculations of Kübler et al. for the $\mathrm{Co}_{2} \mathrm{MnAl}$ and $\mathrm{Co}_{2} \mathrm{MnSn}$ compounds 52. Recently, several groups managed to grow $\mathrm{Co}_{2} \mathrm{MnGe}$ and $\mathrm{Co}_{2} \mathrm{MnSi}$ thin films on various substrates [57, 58, 59, and there also exist first-principles calculations for the (001) surface of such an alloy [60]. Geiersbach and collaborators have grown (110) thin films of $\mathrm{Co}_{2} \mathrm{MnSi}, \mathrm{Co}_{2} \mathrm{MnGe}$ and $\mathrm{Co}_{2} \mathrm{MnSn}$ using a metallic seed on top of a $\mathrm{MgO}(001)$ substrate 61] and studied also the transport properties of multilayers of these compounds with normal metals 62. But as Picozzi et al. have shown the interfaces of such structures are not half-metallic 63. Finally, Kämmerer and collaborators managed to built magnetic tunnel junctions based on $\mathrm{Co}_{2} \mathrm{MnSi}$ and found a tunneling magnetoresistance effect much larger than when the $\mathrm{Ni}_{0.8} \mathrm{Fe}_{0.2}$ or $\mathrm{Co}_{0.3} \mathrm{Fe}_{0.7}$ are used as magnetic electrodes 64. Similar experiments have been undertaken by Inomata and collaborators using $\mathrm{Co}_{2} \mathrm{Cr}_{0.6} \mathrm{Fe}_{0.4} \mathrm{Al}$ as the magnetic electrode 65.

In this contribution, we present a study of the basic electronic and magnetic properties of the half-metallic Heusler alloys. Analyzing the ab-initio results using the group-theory and simple models we explain the origin of the gap in both the half- and full-Heusler alloys, which is fundamental for understanding their electronic and magnetic properties. For both families of compounds the total spin magnetic moment scales with the number of valence electron, thus opening the way to engineer new half-metallic Heusler alloys with the desired magnetic properties. Although in general the surfaces loose the half-metallic character and show only a small degree of spin-polarization, we show that in the case of compounds containing $\mathrm{Cr}$, the very large $\mathrm{Cr}$ moments at the surface reduce the importance of the surface states and the spinpolarization of such surfaces is very high, e.g $84 \%$ for the CrAl-terminated $\mathrm{Co}_{2} \mathrm{CrAl}(001)$ surface. surface. Finally we discuss the role of defects and spinorbit coupling on the half-metallic band gap.

In sections 2 and 3 we present the electronic and magnetic properties of the $\mathrm{XMnSb}(\mathrm{X}=\mathrm{Ni}, \mathrm{Co}, \mathrm{Rh}, \mathrm{Pd}, \mathrm{Ir}$ or $\mathrm{Pt})$ and $\mathrm{Co}_{2} \mathrm{MnZ}(\mathrm{Z}=\mathrm{Al}, \mathrm{Si}, \mathrm{Ga}, \mathrm{Ge}$ or $\mathrm{Sn})$ compounds, respectively. In section 4 we investigate the effect of compressing or expanding the lattice and in section 5 the properties of the quaternary Heusler alloys. In sections [ 6 and 7 we review the role of defects and of the spin-orbit coupling, respectively and finally in section 8 we review the surface properties of these alloys. 


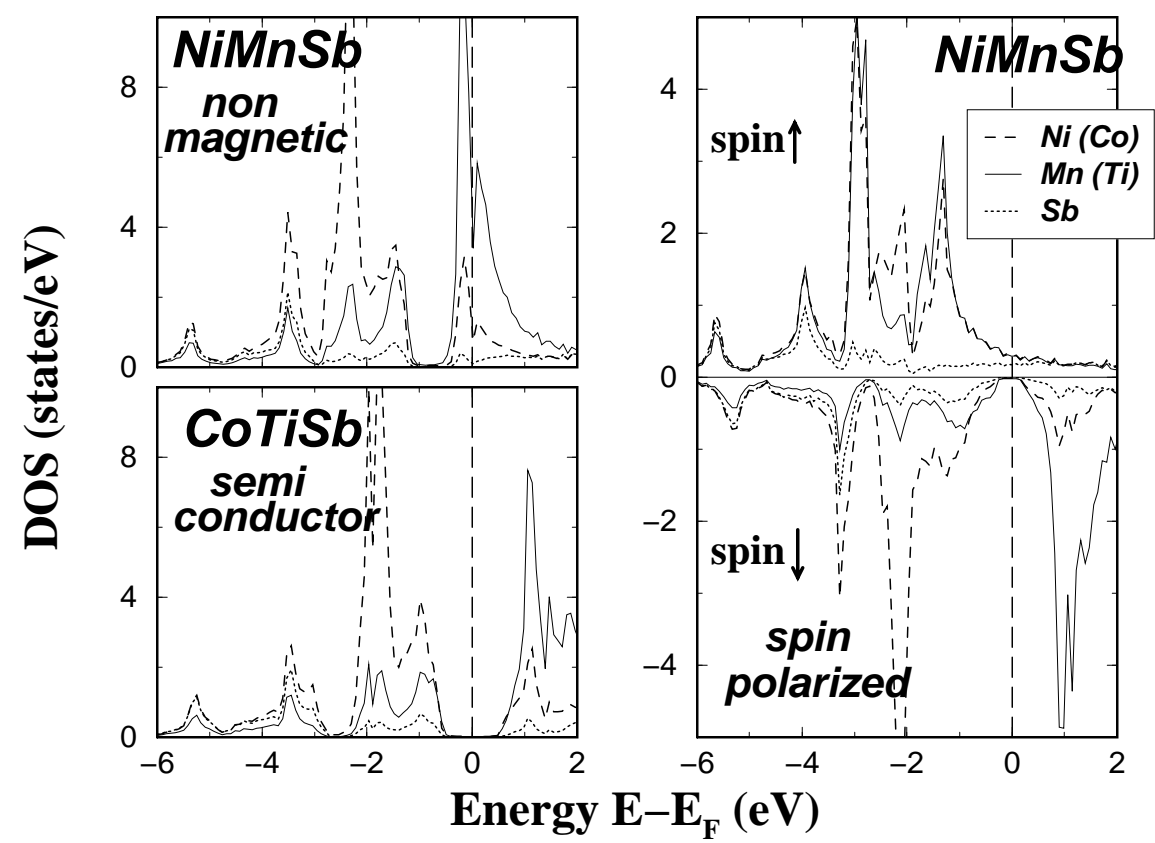

Fig. 3. Atom-resolved density of states (DOS) of NiMnSb for a paramagnetic (left) and ferromagnetic (right) calculation. The zero energy value corresponds to the Fermi level $E_{\mathrm{F}}$

\section{Electronic Structure and Magnetism of Half-Heusler} Alloys

\subsection{Band Structure of Half-Heusler Alloys}

In the following we present results for the densities of states of some typical half-Heusler alloys of $\mathrm{C}_{b}$ structure (see figure 2), sometimes also referred to as semi-Heusler alloys. To perform the calculations, we used the Vosko, Wilk and Nusair parameterization [66 for the local density approximation (LDA) to the exchange-correlation potential [67] to solve the Kohn-Sham equations within the full-potential screened Korringa-Kohn-Rostoker (FSKKR) method 68, 69. The prototype example is NiMnSb, the half-metal discovered in 1983 by de Groot [9. Figure 3 shows the density of states (DOS) of NiMnSb in a non-spin-polarized calculation (left upper panel) and in a calculation correctly including the spin-polarization (right panel). Given are the local contributions to the density of states (LDOS) on the Ni-site (dashed), the Mn-site (full line) and the Sb-site (dotted). In the non-magnetic case the DOS of NiMnSb has contributions from 4 different bands: Each Sb atom with the atomic configuration $5 s^{2} 5 p^{3}$ introduces a deep lying $s$ band, which is located at about $-12 \mathrm{eV}$ and is not shown in the figure, and three $p$-bands in the 
regions between -5.5 and $-3 \mathrm{eV}$. These bands are separated by a deep minimum in the DOS from $5 \mathrm{Ni} d$ bands between -3 and $-1 \mathrm{eV}$, which themselves are separated by a sizeable band gap from the upper $5 d$-bands of Mn. Since all atomic orbitals, i.e. the $\mathrm{Ni} d$, the $\mathrm{Mn} d$ and the $\mathrm{Sb} s p$ orbitals hybridize with each other, all bands are hybrids between these states, being either of bonding or antibonding type. Thus the $\mathrm{Ni} d$-bands contain a bonding $\mathrm{Mn} d$ admixture, while the higher $\mathrm{Mn} d$-bands are antibonding hybrids with small $\mathrm{Ni} d$-admixtures. Equally the Sb $p$-bands exhibit strong Ni $d$ - and somewhat smaller Mn $d$-contributions.

This configuration for NiMnSb is energetically not stable, since (i) the Fermi energy lies in the middle of an antibonding band and (ii) since the Mn atom can gain considerable exchange energy by forming a magnetic moment. Therefore the spin-polarized results (right figure) show a considerably different picture. In the majority ( $\operatorname{spin} \uparrow$ ) band the Mn $d$ states are shifted to lower energies and form a common $d$ band with the $\mathrm{Ni} d$ states, while in the minority band (spin $\downarrow$ ) the Mn states are shifted to higher energies and are unoccupied, so that a band gap at $E_{F}$ is formed separating the occupied $d$ bonding from the unoccupied $d$-type antibonding states. Thus NiMnSb is a half-metal, with a band gap at $E_{F}$ in the minority band and a metallic DOS at $E_{F}$ in the majority band. The total magnetic moment, located mostly at the $\mathrm{Mn}$ atom, can be easily estimated to be exactly $4 \mu_{B}$. Note that NiMnSb has 22 valence electrons per unit cell, 10 from Ni, 7 from $\mathrm{Mn}$ and 5 from $\mathrm{Sb}$. Since, due to the gap at $E_{F}$, in the minority band exactly 9 bands are fully occupied (1 Sb-like $s$ band, 3 Sb-like p bands and 5 Ni-like $d$ bands) and accommodate 9 electrons per unit cell, the majority band contains $22-9=$ 13 electrons, resulting in a moment of $4 \mu_{B}$ per unit cell.

The above non-spinpolarized calculation for NiMnSb (figure 3 left upper panel) suggests, that if we could shift the Fermi energy as in a rigid band model, a particular stable compound would be obtained if the Fermi level falls for both spin directions into the band gap. Then for both spin directions 9 bands would be occupied, resulting in a semiconductor with 18 valence electrons. Such semiconducting Heusler alloys indeed exist. As a typical example, Figure 3 shows also the DOS of CoTiSb, which has a gap of $0.8 \mathrm{eV}[8]$. The gap is indirect corresponding to transitions from the valence band maximum at $\Gamma$ to the conduction band minimum at $X$. Other such semiconductors are CoZrSb $(0.8 \mathrm{eV}), \mathrm{FeVSb}(0.36 \mathrm{eV})$ and NiTiSn $(0.14 \mathrm{eV})$ where the values in the bracket denote the size of the gap $[8]$.

\subsection{XMnSb Half-Heusler Alloys with $\mathrm{X}=\mathrm{Ni}, \mathrm{Pd} \mathrm{Pt}$ and Co, Rh, Ir}

Here we present the electronic structure of the half-Heusler alloys of the type $\mathrm{XMnSb}$, with $\mathrm{X}$ being an element of the Co or Ni columns in the periodic table. These compounds are known experimentally to be ferromagnets with high Curie temperatures ranging between $500 \mathrm{~K}$ and $700 \mathrm{~K}$ for the $\mathrm{Co}, \mathrm{Ni}, \mathrm{Pd}$ 
and Pt compounds, while the Curie temperatures of the Ir and Rh compounds are around room temperature [5. In figure 4 we present the spin-projected total density of states (DOS) for all the six compounds. We remark that all compounds present a gap, which is wider in the compounds containing Co, $\mathrm{Rh}$ or Ir than in $\mathrm{Ni}, \mathrm{Pd}$ or Pt. As above Sb $p$ states occupy the lowest part of the DOS shown in the figure, while the $\mathrm{Sb} s$ states are located $\sim 12 \mathrm{eV}$ below the Fermi level. For the Ni compound the Fermi level is at the middle of the gap and for $\mathrm{PtMnSb}$ at the left edge of the gap in agreement with previous FPLMTO calculations [41]. In the case of CoMnSb the gap is considerably larger $(\sim 1 \mathrm{eV})$ than in the previous two compounds and the Fermi level is located at the left edge of the spin-down gap. CoMnSb has been studied previously by Kübler, who found similar results by using the ASW method. For the other three compounds the Fermi level is located below the gap, although in the case of PdMnSb and IrMnSb it is close to the band edge.

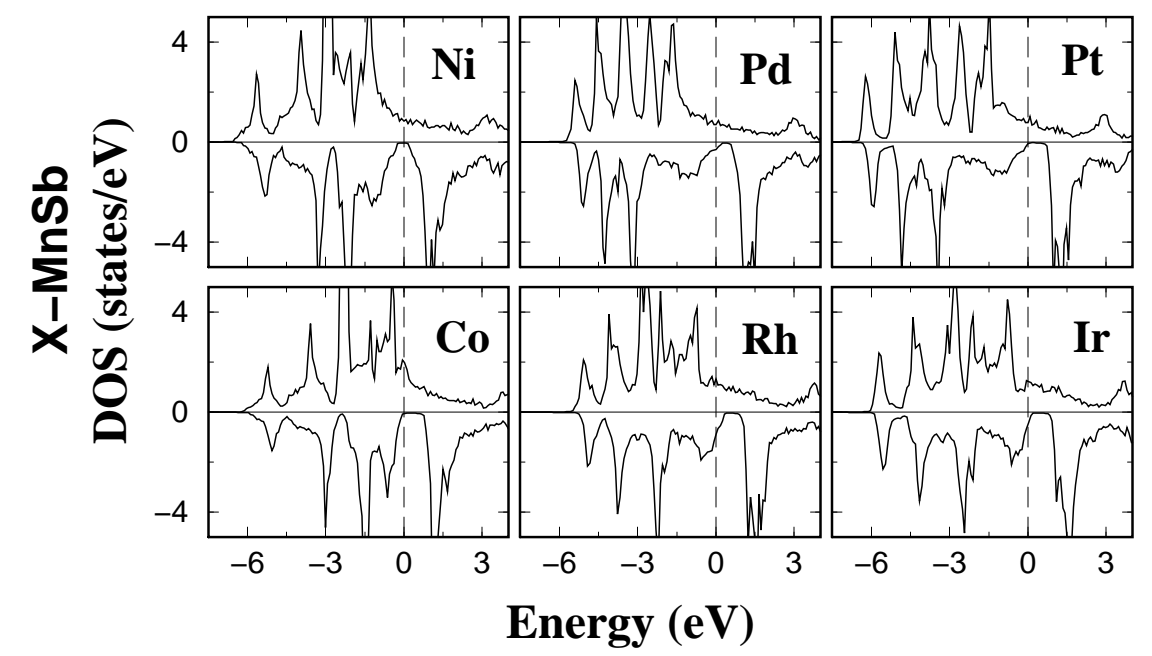

Fig. 4. DOS of $\mathrm{XMnSb}$ compounds for $\mathrm{X}=\mathrm{Ni}, \mathrm{Pd}, \mathrm{Pt}$ and $\mathrm{Co}, \mathrm{Rh}, \mathrm{Pd}$.

The DOS of the different systems are mainly characterized by the large exchange-splitting of the $\mathrm{Mn} d$ states which is around $3 \mathrm{eV}$ in all cases. This leads to large localized spin moments at the Mn site, the existence of which has been verified also experimentally [70. The localization comes from the fact that although $d$ electrons of Mn are itinerant, the spin-down electrons are almost excluded from the Mn site. In table 1 we present the spin magnetic moments at the different sites for all the compounds under study. The moments are calculated by integrating the spin-projected charge density inside every Wigner-Seitz polyhedron. Experimental values for the spin-moment at the Mn site can be deduced from the experiments of Kimura et al. 71] by applying the sum rules to their x-ray magnetic circular dichroism 
spectra and the extracted moments agree nicely with our results; they found a Mn spin moment of $3.85 \mu_{B}$ for NiMnSb, $3.95 \mu_{B}$ for PdMnSb and $4.02 \mu_{B}$ for PtMnSb. In the case of the Co-, Rh-, and IrMnSb compounds the spin magnetic moment of the $\mathrm{X}$ atom is antiparallel to the Mn localized moment and the Mn moment is generally about $0.5 \mu_{B}$ smaller than in the $\mathrm{Ni}, \mathrm{Pd}$ and $\mathrm{Pt}$ compounds. The $\mathrm{Sb}$ atom is here again antiferromagnetically coupled to the Mn atom.

Table 1. Calculated spin magnetic moments in $\mu_{B}$ for the XMnSb compounds. (The experimental lattice constants [5] have been used.)

\begin{tabular}{r|r|r|r|r|r|r}
\hline$m^{\text {spin }}\left(\mu_{B}\right)$ & $\mathrm{X}$ & $\mathrm{Mn}$ & $\mathrm{Sb}$ & \multicolumn{2}{|c}{ Void } & Total \\
\hline NiMnSb & 0.264 & 3.705 & -0.060 & 0.052 & 3.960 \\
$\mathrm{PdMnSb}$ & 0.080 & 4.010 & -0.110 & 0.037 & 4.017 \\
$\mathrm{PtMnSb}$ & 0.092 & 3.889 & -0.081 & 0.039 & 3.938 \\
$\mathrm{CoMnSb}$ & -0.132 & 3.176 & -0.098 & 0.011 & 2.956 \\
$\mathrm{RhMnSb}$ & -0.134 & 3.565 & -0.144 & $<0.001$ & 3.287 \\
$\mathrm{IrMnSb}$ & -0.192 & 3.332 & -0.114 & -0.003 & 3.022 \\
FeMnSb & -0.702 & 2.715 & -0.053 & 0.019 & 1.979 \\
\hline
\end{tabular}

The total magnetic moment in $\mu_{B}$ is just the difference between the number of spin-up occupied states and the spin-down occupied states. As explained above, the number of occupied spin-down states is given by the number of spin down bands, i.e. 9, so that the number of occupied spin-up states is $22-9=13$ for $\mathrm{NiMnSb}$ and the isovalent compounds with $\mathrm{Pd}$ and $\mathrm{Pt}$, but $21-9=12$ for CoMnSb, RhMnSb and IrMnSb and 20-9 = 11 for FeMnSb, provided that the Fermi level stays within the gap. Therefore one expects total moments of $4 \mu_{B}$ for Ni-, Pd- and PtMnSb, $3 \mu_{B}$ for the compounds with $\mathrm{Co}, \mathrm{Rh}$ and Ir and $2 \mu_{B}$ for FeMnSb. In general, for a total number $Z_{t}$ of valence electrons in the unit cell, the total moment $M_{t}$ is given by $M_{t}=Z_{t}-18$, since with 9 electron states occupied in the minority band, $Z_{t}-18$ is just the number of uncompensated electron spins.

The local moment per unit cell as given in table 1 is close to $4 \mu_{B}$ in the case of NiMnSb, PdMnSb and PtMnSb, which is in agreement with the halfmetallic character (or nearly half-metallic character in the case of PdMnSb) observed in figure 4 Note that due to problems with the $\ell_{\max }$ cutoff the KKR method can only give the correct integer number 4, if Lloyd's formula has been used in the evaluation of the integrated density of states, which is not the case in the present calculations. We also find that the local moment of $\mathrm{Mn}$ is not far away from the total number of $4 \mu_{B}$ although there are significant (positive) contributions from the $\mathrm{X}$-atoms and a negative contribution from the $\mathrm{Sb}$ atom. In contrast to this we find that for the half-metallic CoMnSb and IrMnSb compounds the total moment is about $3 \mu_{B}$. Also the local moment of $\mathrm{Mn}$ is reduced, but only by about $0.5 \mu_{B}$. The reduction of the 
total moment to $3 \mu_{B}$ is therefore accompanied by negative Co and Ir spin moments, i.e these atoms couple antiferromagnetically to the Mn moments. The hybridization between $\mathrm{Co}$ and $\mathrm{Mn}$ is considerably larger than between $\mathrm{Ni}$ and Mn being a consequence of the smaller electronegativity difference and the larger extend of the Co orbitals. Therefore the minority valence band of $\mathrm{CoMnSb}$ has a larger $\mathrm{Mn}$ admixture than the one of $\mathrm{NiMnSb}$ whereas the minority conduction band of CoMnSb has a larger Co admixture than the $\mathrm{Ni}$ admixture in the $\mathrm{NiMnSb}$ conduction band, while the populations of the majority bands are barely changed. As a consequence, the Mn moment is reduced by the increasing hybridization, while the Co moment becomes negative, resulting finally in a reduction of the total moment from 4 to $3 \mu_{B}$. The table also shows that further substitution of $\mathrm{Fe}$ for Co leads also to a half-metallic alloy with a total spin magnetic moment of $2 \mu_{B}$ as has been already shown by de Groot et al. in reference [72].

\subsection{Origin of the Gap}

The inspection of the local DOS shown in figure 3 for the ferromagnet NiMnSb as well as for the semiconductor CoTiSb shows that the DOS close to the gap is dominated by $d$-states: in the valence band by bonding hybrids with large $\mathrm{Ni}$ or Co admixture and in the conduction band by the antibonding hybrids with large $\mathrm{Mn}$ or $\mathrm{Ti}$ admixture. Thus the gap originates from the strong hybridization between the $d$ states of the higher valent and the lower valent transition metal atoms. This is shown schematically in figure 5. Therefore the origin of the gap is very similar to the gap in compound semiconductors like GaAs which is enforced by the hybridization of the lower lying As $s p$-states with the energetically higher $\mathrm{Ga} s p$-states. Note that in the $\mathrm{C}_{b}$-structure the $\mathrm{Ni}$ and $\mathrm{Mn}$ sublattices form a zinc-blende structure, which is important for the formation the gap. The difference with respect to GaAs is than only, that $5 d$-orbitals, i.e. $3 t_{2 g}$ and $3 e_{g}$ orbitals, are involved in the hybridization, instead of $4 s p^{3}$-hybrids in the compound semiconductors.

Giving these arguments it is tempting to claim, that also a hypothetical zinc-blende compound like NiMn or PtMn should show a half-metallic character with a gap at $E_{F}$ in the minority band. Figure 6 shows the results of a self-consistent calculation for such zinc-blende NiMn and PtMn, with the same lattice constant as NiMnSb. Indeed a gap is formed in the minority band. In the hypothetical NiMn the Fermi energy is slightly above the gap, however the isoelectronic PtMn compound shows indeed half-metallicity. In this case the occupied minority bands consists of six bands, a low-lying $s$ band and five bonding $d$-bands, both of mostly Pt character. Since the total number of valence electrons is 17 , the majority bands contain 11 electrons, so that the total moment per unit cell is $11-6=5 \mu_{B}$, which is indeed obtained in the calculations. This is the largest possible moment for this compound, since in the minority band all $5 \mathrm{Mn} d$-states are empty while all majority 


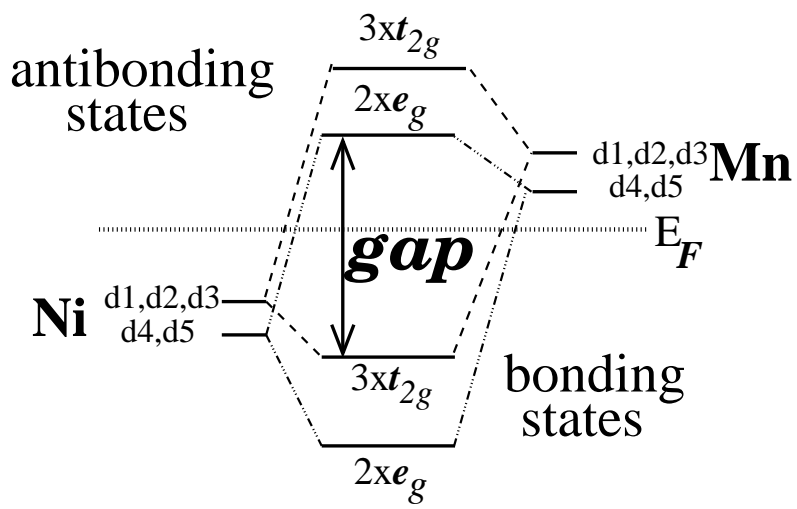

Fig. 5. Schematic illustration of the origin of the gap in the minority band in half-Heusler alloys and in compound semiconductors: The energy levels $E_{b}$ of the energetically lower lying bonding hybrids are separated from the levels $E_{a b}$ of the antibonding hybrids by a gap, such that only the bonding states are occupied.

$d$-states are occupied. The same limit of $5 \mu_{B}$ is also the maximal possible moment of the half-metallic $C 1_{b}$ Heusler alloys.

The gap in the half-metallic $C 1_{b}$ compounds is normally an indirect gap, with the maximum of the valence band at the $\Gamma$ point and the minimum of the conduction band at the $X$-point. For NiMnSb we obtain a band gap of about $0.5 \mathrm{eV}$, which is in good agreement with the experiments of Kirillova and collaborators [29], who, analyzing their infrared spectra, estimated a gap width of $\sim 0.4 \mathrm{eV}$. As seen already from figure 4 the gap of $\mathrm{CoMnSb}$ is considerable larger $(\sim 1 \mathrm{eV})$ and the Fermi level is located at the edge of the minority valence band.

As it is well-known, the local density approximation (LDA) and the generalized gradient approximation (GGA) strongly underestimate the values of the gaps in semiconductors, typically by a factor of two. However, very good values for these gaps are obtained in the so-called GW approximation of Hedin and Lundqvist 73, which describes potential in semiconductors very well. On the other hand the minority gap in the half-metallic systems might be better described by the LDA and GGA since in these system the screening is metallic.

\subsection{Role of $s p$-Elements}

While the $s p$-elements are not responsible for the existence of the minority gap, they are nevertheless very important for the physical properties of the Heusler alloys and the structural stability of the $C 1_{b}$ structure, as we discuss in the following.

While an $\mathrm{Sb}$ atom has 5 valence electrons $\left(5 s^{2}, 5 p^{3}\right)$, in the NiMnSb compound each Sb atom introduces a deep lying $s$-band, at about $-12 \mathrm{eV}$, 


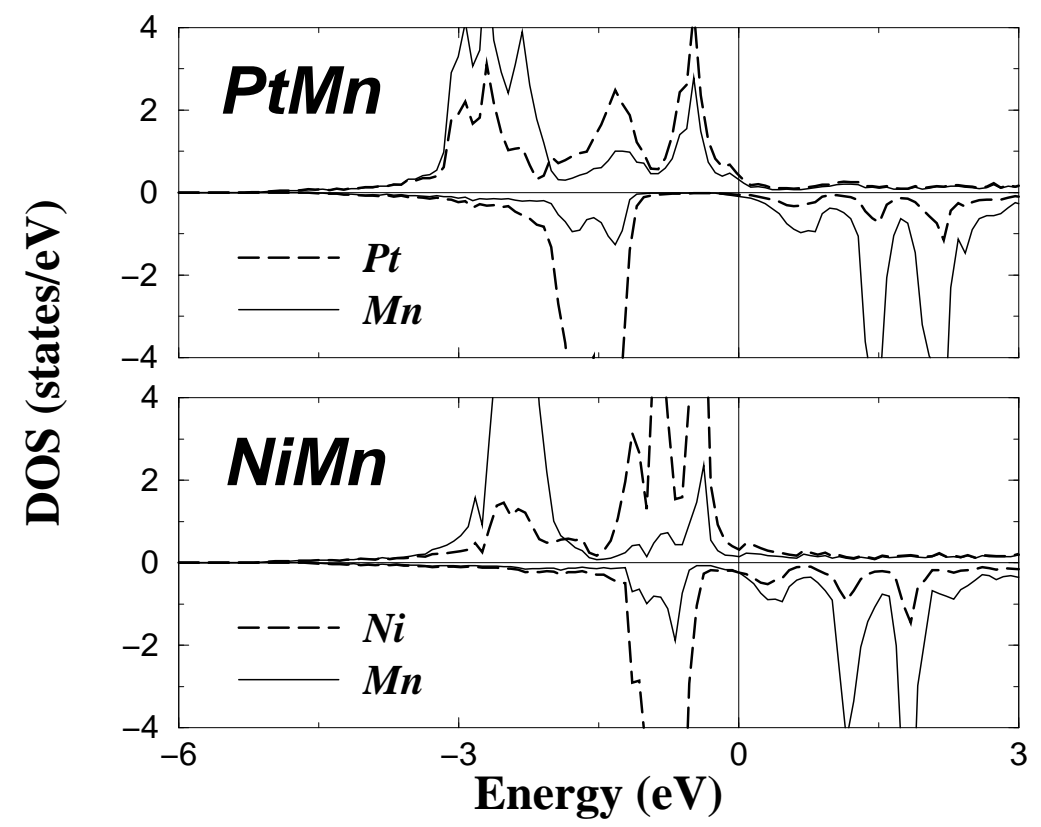

Fig. 6. Atom-resolved DOS for the hypothetical PtMn and NiMn crystallizing in the zinc-blende structure.

and three $p$-bands below the center of the $d$-bands. These bands accommodate a total of 8 electrons per unit cell, so that formally $\mathrm{Sb}$ acts as a triple charged $\mathrm{Sb}^{-3}$ ion. Analogously, a Te-atom behaves in these compounds as a $\mathrm{Te}^{-2}$ ion and a Sn-atom as a $\mathrm{Sn}^{-4}$ ion. This does not mean, that locally such a large charge transfer exists. In fact, the $s$ - and $p$-states strongly hybridize with the TM $d$-states and the charge in these bands is delocalized and locally Sb even looses about one electron, if one counts the charge in the Wigner-Seitz cells. What counts is that the $s$ - and $p$-bands accommodate 8 electrons per unit cell, thus effectively reducing the $d$-charge of the TM atoms.

This is nicely illustrated by the existence of the semiconducting compounds CoTiSb and NiTiSn. Compared to CoTiSb, in NiTiSn the missing $p$-charge of the Sn atom is replace by an increased $d$ charge of the Ni atom, so that in both cases all 9 valence bands are occupied.

The $s p$-atom is very important for the structural stability of the Heusler alloys. For instance, it is difficult to imagine that the calculated half-metallic NiMn and PtMn alloys with zinc-blende structure, the LDOS of which are shown in figure [ actually exist, since metallic alloys prefer high coordinated structures like fcc, bcc, hcp etc. Therefore the $s p$-elements are decisive of the stability of the $C 1_{b}$ compounds. A careful discussion of the bonding in these compounds has been recently published by Nanda and Dasgupta [74] 
using the crystal orbital Hamiltonian population (COHP) method. For the semiconductor FeVSb they find that while the largest contribution to the bonding arises from the V- $d-\mathrm{Fe}-d$ hybridization, contributions of similar size arise also from the Fe- $d$ - Sb- $p$ and the V- $d-$ Sb- $p$ hybridization. Similar results are also valid for the semiconductors like CoTiSb and NiTiSn and in particular for the half-metal NiMnSb. Since the majority $d$-band is completely filled, the major part of the bonding arises from the minority band, so that similar arguments as for the semiconductors apply.

Another property of the $s p$-elements is worthwhile to mention: substituting the $\mathrm{Sb}$ atom in NiMnSb by $\mathrm{Sn}$, In or Te destroys the half-metallicity 10. This is in contrast to the substitution of $\mathrm{Ni}$ by $\mathrm{Co}$ or Fe, which is documented in table 1 The total moment of $4 \mu_{B}$ for $\mathrm{NiMnSb}$ is reduced to $3 \mu_{B}$ in CoMnSb and $2 \mu_{B}$ in FeMnSb, thus preserving half-metallicity. In NiMnSn the total moment is reduced to $3.3 \mu_{B}$ (instead of 3) and in NiMnTe the total moment increases only to $4.7 \mu_{B}$ (instead of 5 ). Thus by changing the $s p$-element it is rather difficult to preserve the half-metallicity, since the density of states changes more like in a rigid band model [10].

\subsection{Slater-Pauling Behavior}

As discussed above the total moment of the half-metallic $C 1_{b}$ Heusler alloys follows the simple rule: $M_{t}=Z_{t}-18$, where $Z_{t}$ is the total number of valence electrons. In short, the total number of electrons $Z_{t}$ is given by the sum of the number of spin-up and spin-down electrons, while the total moment $M_{t}$ is given by the difference

$$
Z_{t}=N_{\uparrow}+N_{\downarrow} \quad, \quad M_{t}=N_{\uparrow}-N_{\downarrow} \quad \rightarrow \quad M_{t}=Z_{t}-2 N_{\downarrow}
$$

Since 9 minority bands are fully occupied, we obtain the simple "rule of 18 " for half-metallicity in $C 1_{b}$ Heusler alloys

$$
M_{t}=Z_{t}-18
$$

the importance of which has been recently pointed out by Jung et al. [75] and Galanakis et al. [10. It is a direct analogue to the well-known Slater-Pauling behavior of the binary transition metal alloys [76. The difference with respect to these alloys is, that in the half-Heusler alloys the minority population is fixed to 9 , so that the screening is achieved by filling the majority band, while in the transition metal alloys the majority band is filled with $5 d$-states and charge neutrality is achieved by filling the minority states. Therefore in the TM alloys the total moment is given by $M_{t}=10-Z_{t}$. Similar rules with integer total moments are also valid for other half-metals, e.g. for the fullHeusler alloys like $\mathrm{Co}_{2} \mathrm{MnGe}$ with $L 2_{1}$ structure. For these alloys we will in section 3 derive the "rule of 24 ": $M_{t}=Z_{t}-24$, with arises from the fact that the minority band contains 12 electrons. For the half-metallic zinc-blende compounds like CrAs the rule is: $M_{t}=Z_{t}-8$, since the minority As-like 
valence bands accommodate 4 electrons 17. In all cases the moments are integer.

In figure 7 we have gathered the calculated total spin magnetic moments for the half-Heusler alloys which we have plotted as a function of the total number of valence electrons. The dashed line represents the rule $M_{t}=Z_{t}-18$ obeyed by these compounds. The total moment $M_{t}$ is an integer quantity, assuming the values $0,1,2,3,4$ and 5 if $Z_{t} \geq 18$. The value 0 corresponds to the semiconducting phase and the value 5 to the maximal moment when all 10 majority $d$-states are filled. Firstly we varied the valence of the lowervalent (i.e. magnetic) transition metal atom. Thus we substitute $\mathrm{V}, \mathrm{Cr}$ and $\mathrm{Fe}$ for $\mathrm{Mn}$ in the NiMnSb and CoMnSb compounds using the experimental lattice constants of the two Mn compounds. For all these compounds we find that the total spin moment scales accurately with the total charge and that they all present the half-metallicity.

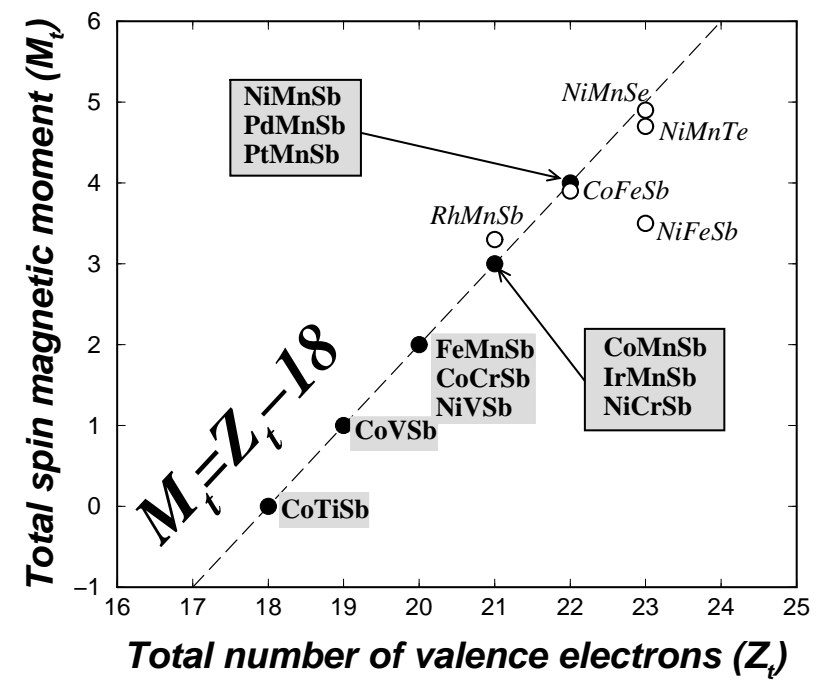

Fig. 7. Calculated total spin moments for all the studied half Heusler alloys. The dashed line represents the Slater-Pauling behavior. With open circles we present the compounds deviating from the SP curve.

As a next test we have substituted Fe for $\mathrm{Mn}$ in CoMnSb and NiMnSb, but both $\mathrm{CoFeSb}$ and $\mathrm{NiFeSb}$ loose their half-metallic character. In the case of NiFeSb the majority $d$-states are already fully occupied as in NiMnSb, thus the additional electron has to be screened by the minority $d$-states, so that the Fermi level falls into the minority Fe states and the half-metallicity is lost; for half-metallicity a total moment of $5 \mu_{B}$ would be required which is clearly not possible. For CoFeSb the situation is more delicate. This system has 22 valence electrons and if it would be a half-metal, it should have a total 
spin-moment of $4 \mu_{B}$ as NiMnSb. In reality our calculations indicate that the Fermi level is slightly above the gap and the total spin-moment is slightly smaller than $4 \mu_{B}$. The Fe atom possesses a comparable spin-moment in both $\mathrm{NiFeSb}$ and $\mathrm{CoFeSb}$ compounds contrary to the behavior of the $\mathrm{V}, \mathrm{Cr}$ and $\mathrm{Mn}$ atoms. Except $\mathrm{NiFeSb}$ other possible compounds with 23 valence electrons are NiMnTe and NiMnSe. We have calculated their magnetic properties using the lattice constant of NiMnSb. As shown in figure [7 NiMnSe almost makes the $5 \mu_{B}$ (its total spin moment is $4.86 \mu_{B}$ ) and is nearly half-metallic, while its isovalent, NiMnTe, has a slightly smaller spin moment. NiMnSe and NiMnTe show big changes in the majority band compared to systems with 22 valence electrons as $\mathrm{NiMnSb}$ or NiMnAs, since antibonding $p$ - $d$ states, which are usually above $E_{F}$, are shifted below the Fermi level, thus increasing the total moment to nearly $5 \mu_{B}$.

\section{Full Heusler Alloys}

\subsection{Electronic Structure of $\mathrm{Co}_{2} \mathrm{MnZ}$ with $\mathrm{Z}=\mathrm{Al}$, Si, Ga, Ge and Sn}

The second family of Heusler alloys, which we discuss, are the full-Heusler alloys. We consider in particular compounds containing Co and Mn, as these are the full-Heusler alloys that have attracted most of the attention. They are all strong ferromagnets with high Curie temperatures (above $600 \mathrm{~K}$ ) and except the $\mathrm{Co}_{2} \mathrm{MnAl}$ they show very little disorder [5]. They adopt the $L 2_{1}$ structure shown in figure 2 Each Mn or $s p$ atom has eight Co atoms as first neighbors, sitting in an octahedral symmetry position, while each Co has four Mn and four $s p$ atoms as first neighbors and thus the symmetry of the crystal is reduced to the tetrahedral one. The Co atoms occupying the two different sublattices are chemically equivalent as the environment of the one sublattice is the same as the environment of the second one but rotated by $90^{\circ}$. The occupancy of two fcc sublattices by Co (or in general by $\mathrm{X}$ ) atoms distinguish the full-Heusler alloys with the $L 2_{1}$ structure from the half-Heusler compounds with the $C 1_{b}$ structure, like e.g. CoMnSb, where only one sublattice is occupied by Co atoms and the other one is empty. Although in the $L 2_{1}$ structure, the Co atoms are sitting on second neighbor positions, their interaction is important to explain the magnetic properties of these compounds as we will show in the next section.

In figure 8 we have gathered the spin-resolved density of states (DOS) for the $\mathrm{Co}_{2} \mathrm{MnAl}, \mathrm{Co}_{2} \mathrm{MnGa}, \mathrm{Co}_{2} \mathrm{MnSi}$ and $\mathrm{Co}_{2} \mathrm{MnGe}$ compounds calculated using the FSKKR. Firstly as shown by photoemission experiments by Brown et al. in the case of $\mathrm{Co}_{2} \mathrm{MnSn}$ [77 and verified by our calculations, the valence band extends around $6 \mathrm{eV}$ below the Fermi level and the spin-up DOS shows a large peak just below the Fermi level for these compounds. Although Ishida et al. 53. predicted them to be half-metals with small spin-down gaps 


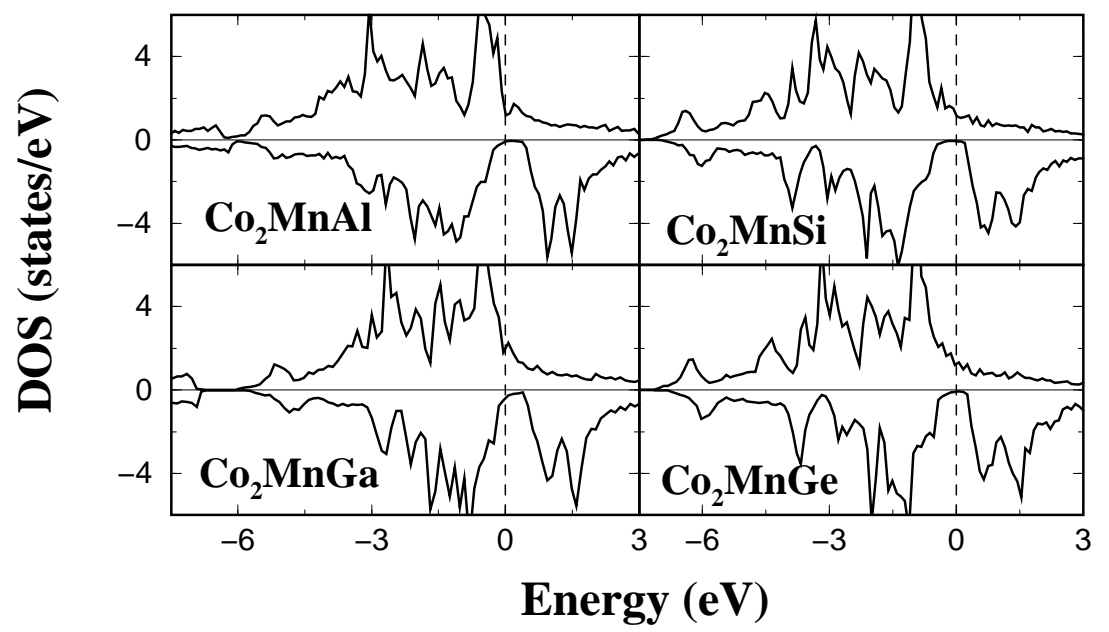

Fig. 8. Atom-resolved DOS for the $\mathrm{Co}_{2} \mathrm{MnZ}$ compounds with $\mathrm{Z}=\mathrm{Al}$, $\mathrm{Si}, \mathrm{Ge}, \mathrm{Sn}$ compounds

ranging from 0.1 to $0.3 \mathrm{eV}$ depending on the material, our previous calculations showed a very small DOS at the Fermi level, in agreement with the ASW results of Kübler et al. [76] for $\mathrm{Co}_{2} \mathrm{MnAl}$ and $\mathrm{Co}_{2} \mathrm{MnSn}$. However a recalculation of our KKR results with a higher $\ell$-cut-off of $\ell_{\max }=4$ restores the gap and we obtain good agreement with the recent results of Picozzi et al. using the FLAPW method. The gap is an indirect gap, with the maximum of the valence band at $\Gamma$ and the minimum of the conduction band at the $X$-point.

In the case of the half-Heusler alloys like NiMnSb the Mn spin magnetic moment is very localized due to the exclusion of the spin-down electrons at the Mn site and amounts to about $3.7 \mu_{B}$ in the case of NiMnSb. In the case of CoMnSb the increased hybridization between the Co and Mn spindown electrons decreased the Mn spin moment to about $3.2 \mu_{B}$ (in table 2 we have gathered the atomic-resolved and total moments of the $\mathrm{Co}_{2} \mathrm{MnZ}$ compounds). In the case of the full-Heusler alloys each Mn atom has eight Co atoms as first neighbors instead of four as in CoMnSb and the above hybridization is very important decreasing even further the Mn spin moment to less than $3 \mu_{B}$ except in the case of $\mathrm{Co}_{2} \mathrm{MnSn}$ where it is comparable to the $\mathrm{CoMnSb}$ compound. The Co atoms are ferromagnetically coupled to the Mn spin moments and they posses a spin moment that varies from $\sim 0.7$ to 1.0 $\mu_{B}$. Note that in the half-metallic $C 1_{b}$ Heusler alloys, the $X$-atom has a very small moment only, in the case of $\mathrm{CoMnSb}$ the Co moment is even negative. However in the full Heusler alloys the Co moment is large and positive and arises basically from two unoccupied Co bands in the minority conduction band, as explained below. Therefore both Co atoms together can have a 
moment of about $2 \mu_{B}$, if all majority Co states are occupied. This is basically the case for $\mathrm{Co}_{2} \mathrm{MnSi}, \mathrm{Co}_{2} \mathrm{MnGe}$ and $\mathrm{Co}_{2} \mathrm{MnSn}$ (see table 2). In contrast to this the $s p$ atom has a very small negative moment which is one order of magnitude smaller than the Co moment. The negative sign of the induced $s p$ moment characterizes most of the studied full and half Heusler alloys with very few exceptions. The compounds containing $\mathrm{Al}$ and $\mathrm{Ga}$ have 28 valence electrons and the ones containing $\mathrm{Si}, \mathrm{Ge}$ and $\mathrm{Sn} 29$ valence electrons. The first compounds have a total spin moment of $4 \mu_{B}$ and the second ones of 5 $\mu_{B}$ which agree with the experimental deduced moments of these compounds [78. So it seems that the total spin moment, $M_{t}$, is related to the total number of valence electrons, $Z_{t}$, by the simple relation: $M_{t}=Z_{t}-24$, while in the half-Heusler alloys the total magnetic moment is given by the relation $M_{t}=Z_{t}-18$. In the following section we will analyze the origin of this rule.

Table 2. Calculated spin magnetic moments in $\mu_{B}$ using the experimental lattice constants (see reference [5]) for the $\mathrm{Co}_{2} \mathrm{MnZ}$ compounds, where $\mathrm{Z}$ stands for the $s p$ atom.

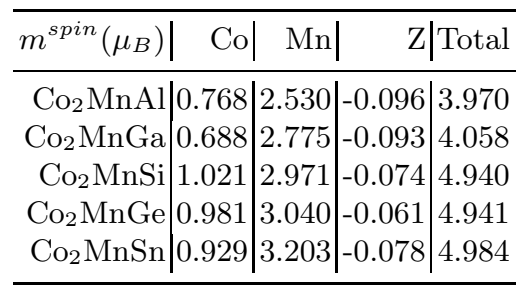

\subsection{Origin of the gap in Full-Heusler Alloys}

Since, similar to the half-Heusler alloys, the four $s p$-bands are located far below the Fermi level and thus are not relevant for the gap, we consider only the hybridization of the $15 d$ states of the Mn atom and the two Co atoms. For simplicity we consider only the $d$-states at the $\Gamma$ point, which show the full structural symmetry. We will give here a qualitative picture, since a through group theoretical analysis has been given in reference [11]. Note that the Co atoms form a simple cubic lattice and that the Mn atoms (and the Ge atoms) occupy the body centered sites and have 8 Co atoms as nearest neighbors. Although the distance between the Co atoms is a second neighbor distance, the hybridization between these atoms is qualitatively very important. Therefore we start with the hybridization between these Co atoms which is qualitatively sketched in figure 9 The $5 d$-orbitals are divided into the twofold degenerate $d_{4}, d_{5}\left(r^{2}, x^{2}-y^{2}\right)$ and the threefold degenerate $d_{1}$, $d_{2}, d_{3}(x y, y z, z x)$ states. The $e_{g}$ orbitals $\left(t_{2 g}\right.$ orbitals) can only couple with the $e_{g}$ orbitals ( $t_{2 g}$ orbitals) of the other Co atom forming bonding hybrids, 
denoted by $e_{g}$ (or $t_{2 g}$ ) and antibonding orbitals, denoted by $e_{u}$ (or $t_{1 u}$ ). The coefficients in front of the orbitals give the degeneracy.

In a second step we consider the hybridization of these Co-Co orbitals with the Mn $d$-orbitals. As we show in the second part of figure 9 the double degenerated $e_{g}$ orbitals hybridize with the $d_{4}$ and $d_{5}$ of the Mn that transform also with the same representation. They create a double degenerated bonding $e_{g}$ state that is very low in energy and an antibonding one that is unoccupied and above the Fermi level. The $3 \times t_{2 g}$ Co orbitals couple to the $d_{1,2,3}$ of the Mn and create 6 new orbitals, 3 of which are bonding and are occupied and the other three are antibonding and high in energy. Finally the $2 \times e_{u}$ and $3 \times t_{1 u}$ Co orbitals can not couple with any of the Mn $d$-orbitals since none of these is transforming with the $u$ representations and they are orthogonal to the Co $e_{u}$ and $t_{1 u}$ states. With respect to the Mn and the Ge atoms these states are therefore non-bonding. The $t_{1 u}$ states are below the Fermi level and they are occupied while the $e_{u}$ are just above the Fermi level. Thus in total 8 minority $d$-bands are filled and 7 are empty.

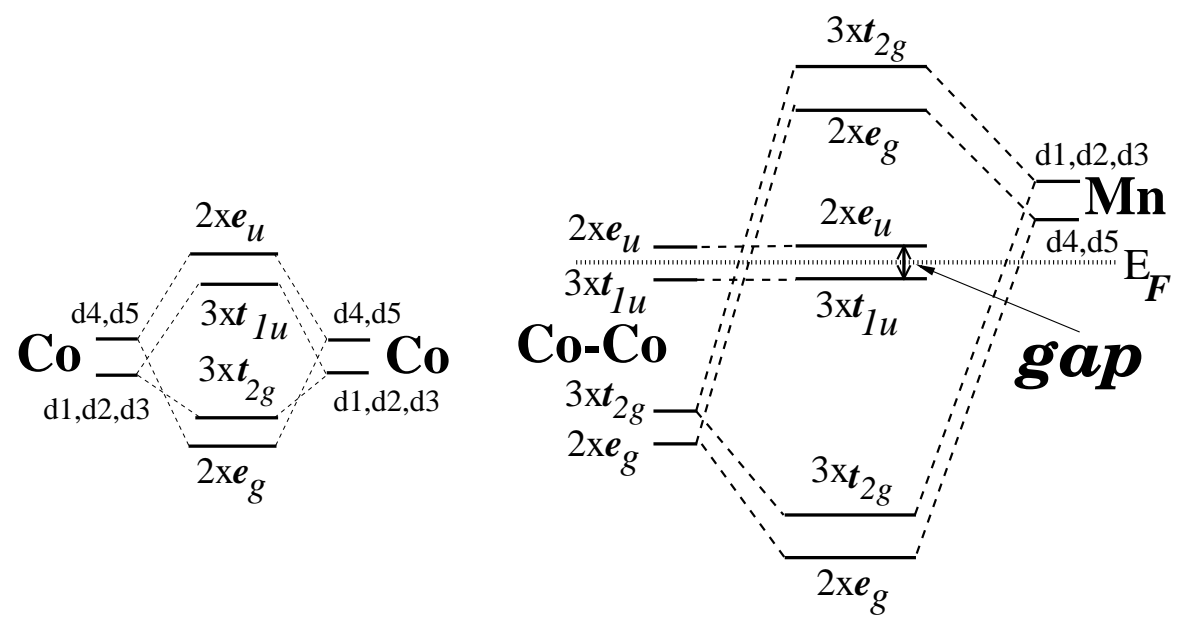

Fig. 9. Schematic illustration of the origin of the gap in the minority band in full-Heusler alloys.

Therefore all 5 Co-Mn bonding bands are occupied and all 5 Co-Mn antibonding bands are empty, and the Fermi level falls in between the 5 nonbonding Co bands, such that the three $t_{1 u}$ bands are occupied and the two $e_{u}$ bands are empty. The maximal moment of the full Heusler alloys is therefore $7 \mu_{B}$ per unit cell, which is achieved, if all majority $d$-states are occupied.

In order to demonstrate the existence of the $t_{1 u}$ and $e_{u}$ states at the Fermi level, we show in figure 10 the LDOS of $\mathrm{Co}_{2} \mathrm{MnGe}$ at the Co and Mn sites, which are splitted up into the local $d_{1}, d_{2}, d_{3}$ orbitals (normally referred to as $t_{2 g}$; full lines) and the local $d_{4}, d_{5}$ orbitals (normally $e_{g}$; dashed). In the 


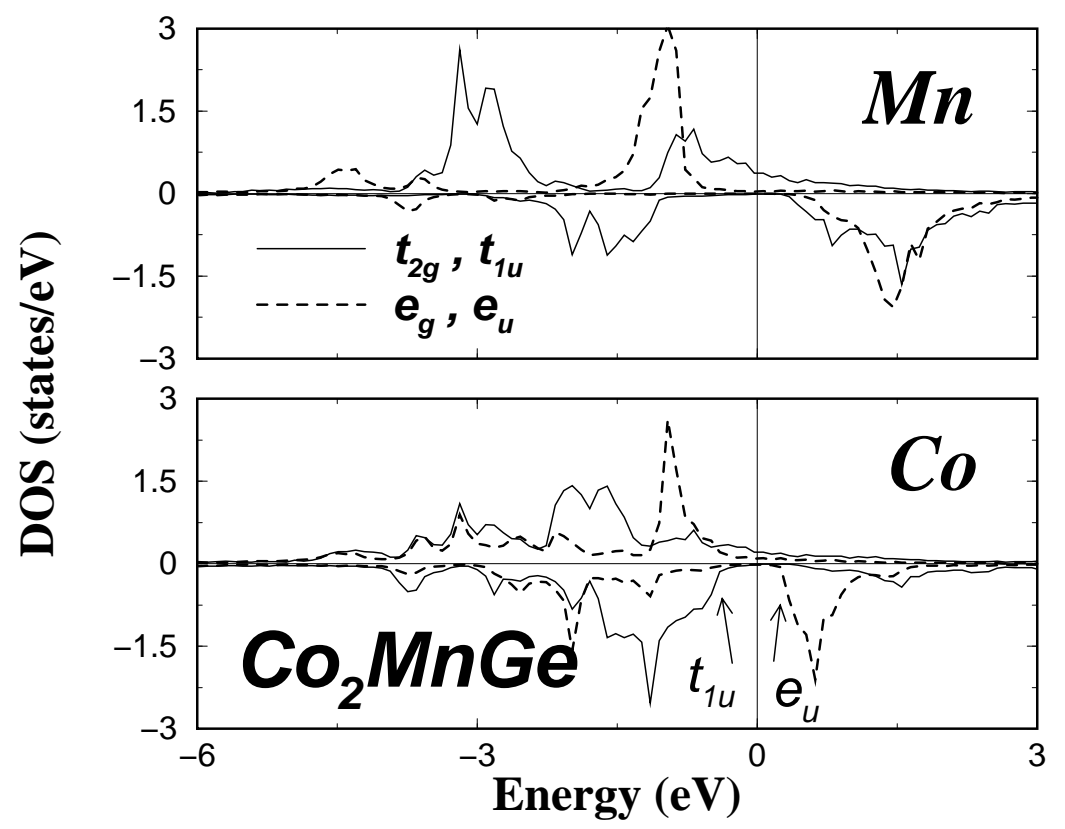

Fig. 10. Atom- and angular momentum - resolved DOS for the $\mathrm{Co}_{2} \mathrm{MnGe}$ compound.

nomenclature used above, the $d_{1}, d_{2}, d_{3}$ contributions contain both the $t_{2 g}$ and the $t_{1 u}$ contributions, while the $d_{4}, d_{5}$ orbitals contain the $e_{g}$ and $e_{u}$ contributions. The Mn DOS clearly shows a much bigger effective gap at $E_{F}$, considerably larger than in $\mathrm{CoMnSb}$ (figure 4), as one would expect from the stronger hybridization in $\mathrm{Co}_{2} \mathrm{MnGe}$. However the real gap is determined by the Co-Co interaction only, in fact by the $t_{1 u}-e_{u}$ splitting, and is smaller than in CoMnSb. Thus the origin of the gap in the full-Heusler alloys is rather subtle.

\subsection{Slater-Pauling behavior of the Full-Heusler alloys}

Following the above discussion we will investigate the Slater-Pauling behavior and in figure 11 we have plotted the total spin magnetic moments for all the compounds under study as a function of the total number of valence electrons. The dashed line represents the half metallicity rule: $M_{t}=Z_{t}-24$ of the full Heusler alloys. This rule arises from the fact that the minority band contains 12 electrons per unit cell: 4 are occupying the low lying $s$ and $p$ bands of the $s p$ element and 8 the Co-like minority $d$ bands $\left(2 \times e_{g}, 3 \times t_{2 g}\right.$ and $\left.3 \times t_{1 u}\right)$, as explained above (see figure 9). Since 7 minority bands are unoccupied, the largest possible moment is $7 \mu_{B}$ and occurs when all majority $d$-states are occupied. 
Overall we see that many of our results coincide with the Slater-Pauling curve. Some of the Rh compounds show small deviations which are more serious for the $\mathrm{Co}_{2} \mathrm{TiAl}$ compound. We see that there is no compound with a total spin moment of $7 \mu_{B}$ or even $6 \mu_{B}$. Moreover we found also examples of half-metallic materials with less than 24 electrons, $\mathrm{Mn}_{2}$ VGe with 23 valence electrons and $\mathrm{Mn}_{2} \mathrm{VAl}$ with 22 valence electrons. Firstly, we have calculated the spin moments of the compounds $\mathrm{Co}_{2} \mathrm{YAl}$ where $\mathrm{Y}=\mathrm{Ti}, \mathrm{V}, \mathrm{Cr}, \mathrm{Mn}$ and Fe. The compounds containing $\mathrm{V}, \mathrm{Cr}$ and $\mathrm{Mn}$ show a similar behavior. As we substitute $\mathrm{Cr}$ for $\mathrm{Mn}$, which has one valence electron less than $\mathrm{Mn}$, we depopulate one $\mathrm{Mn}$ spin-up state and thus the spin moment of $\mathrm{Cr}$ is around $1 \mu_{B}$ smaller than the $\mathrm{Mn}$ one while the Co moments are practically the same for both compounds. Substituting V for Cr has a larger effect since also the Co spin-up DOS changes slightly and the Co magnetic moment is increased by about $0.1 \mu_{B}$ compared to the other two compounds and $\mathrm{V}$ possesses a small moment of $0.2 \mu_{B}$. This change in the behavior is due to the smaller hybridization between the Co atoms and the $\mathrm{V}$ ones as compared to the $\mathrm{Cr}$ and $\mathrm{Mn}$ atoms. Although all three $\mathrm{Co}_{2} \mathrm{VAl}, \mathrm{Co}_{2} \mathrm{CrAl}$ and $\mathrm{Co}_{2} \mathrm{MnAl}$ compounds are on the SP curve as can be seen in figure 11] this is not the case for the compounds containing Fe and Ti. If the substitution of Fe for Mn followed the same logic as the one of $\mathrm{Cr}$ for $\mathrm{Mn}$ then the Fe moment should be around $3.5 \mu_{B}$ which is a very large moment for the Fe site. Therefore it is energetically more favorable for the system that also the Co moment is increased, as it was also the case for the other systems with 29 electrons like $\mathrm{Co}_{2} \mathrm{MnSi}$, but while the latter one makes it to $5 \mu_{B}, \mathrm{Co}_{2} \mathrm{FeAl}$ reaches a value of $4.9 \mu_{B}$. In the case of $\mathrm{Co}_{2} \mathrm{TiAl}$, it is energetically more favorable to have a weak ferromagnet than an integer moment of $1 \mu_{B}$ as it is very difficult to magnetize the Ti atom. Even in the case of the $\mathrm{Co}_{2} \mathrm{TiSn}$ the calculated total spin magnetic moment of $1.78 \mu_{B}$ (compared to the experimental value of $1.96 \mu_{B}[79]$ ) arises only from the Co atoms as was also shown experimentally by Pendl et al. [80, and the Ti atom is practically nonmagnetic and the latter compound fails to follow the SP curve.

As a second family of materials we have studied the compounds containing $\mathrm{Fe}$. $\mathrm{Fe}_{2} \mathrm{VAl}$ has in total 24 valence electrons and is a semi-metal, i.e. nonmagnetic with a very small DOS at the Fermi level, as it is already known experimentally [81]. All the studied Fe compounds follow the SP behavior as can be seen in figure 11] In the case of the $\mathrm{Fe}_{2} \mathrm{CrAl}$ and $\mathrm{Fe}_{2} \mathrm{MnAl}$ compounds the $\mathrm{Cr}$ and $\mathrm{Mn}$ atoms have spin moments comparable to the Co compounds and similar DOS. In order to follow the $\mathrm{SP}$ curve the $\mathrm{Fe}$ in $\mathrm{Fe}_{2} \mathrm{CrAl}$ is practically nonmagnetic while in $\mathrm{Fe}_{2} \mathrm{MnAl}$ it has a small negative moment. When we substitute $\mathrm{Si}$ for $\mathrm{Al}$ in $\mathrm{Fe}_{2} \mathrm{MnAl}$, the extra electron exclusively populates Fe spin-up states and the spin moment of each Fe atom is increased by 0.5 $\mu_{B}$ contrary to the corresponding Co compounds where also the Mn spin moment was considerably increased. Finally we calculated as a test $\mathrm{Mn}_{2} \mathrm{VAl}$ and $\mathrm{Mn}_{2} \mathrm{VGe}$ that have 22 and 23 valence electrons, respectively, to see if we can 


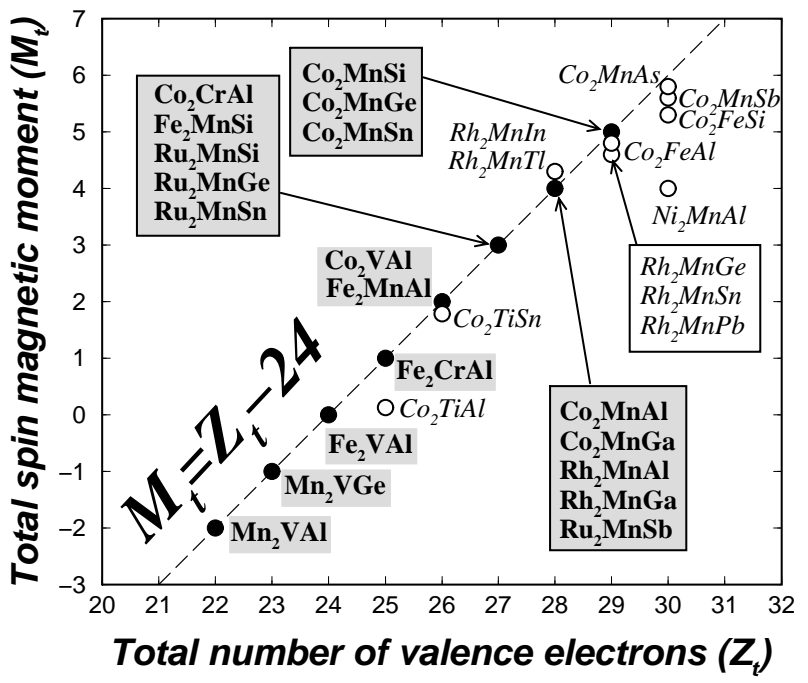

Fig. 11. Calculated total spin moments for all the studied full Heusler alloys. The dashed line represents the Slater-Pauling behavior. With open circles we present the compounds deviating from the SP curve.

reproduce the SP behavior not only for compounds with more than 24, but also for compounds with less than 24 electrons. As we have already shown $\mathrm{Fe}_{2} \mathrm{VAl}$ is nonmagnetic and $\mathrm{Co}_{2} \mathrm{VAl}$, which has two electrons more, has a spin moment of $2 \mu_{B} . \mathrm{Mn}_{2} \mathrm{VAl}$ has two valence electrons less than $\mathrm{Fe}_{2} \mathrm{VAl}$ and its total spin moment is $-2 \mu_{B}$ and thus it follows the SP behavior; negative total spin moment means that the "minority" band with the gap has more occupied states than the "majority" one.

As we have already mentioned the maximal moment of a full-Heusler alloy is seven $\mu_{B}$, and should occur, when all 15 majority $d$ states are occupied. Analogously for a half-Heusler alloy the maximal moment is $5 \mu_{B}$. However this limit is difficult to achieve, since due to the hybridization of the $d$ states with empty $s p$-states of the transition metal atoms (sites $\mathrm{X}$ and $\mathrm{Y}$ in figure 2), $d$-intensity is transferred into states high above $E_{F}$, which are very difficult to occupy. Although in the case of half-Heusler alloys, we could identify systems with a moment of nearly $5 \mu_{B}$, the hybridization is much stronger in the full-Heusler alloys so that a total moment of $7 \mu_{B}$ seems to be impossible. Therefore we restrict our search to possible systems with $6 \mu_{B}$, i.e. systems with 30 valence electrons, but as shown also in figure [1] none of them makes exactly the $6 \mu_{B}$. $\mathrm{Co}_{2} \mathrm{MnAs}$ shows the largest spin moment: $5.8 \mu_{B}$. The basic reason, why moments of $6 \mu_{B}$ are so difficult to achieve, is that as a result of the strong hybridization with the two Co atoms the Mn atom cannot have a much larger moment than $3 \mu_{B}$. While due to the empty $e_{u}$-states the two 
Co atoms have no problem to contribute a total of $2 \mu_{B}$, the Mn moment is hybridization limited.

\section{Effect of the Lattice Parameter}

In this section we will study the influence of the lattice parameter on the electronic and magnetic properties of the $C 1_{b}$ and $L 2_{1}$ Heusler alloys. For these reason we plot in figure 12 the $\mathrm{DOS}$ of $\mathrm{NiMnSb}$ and $\mathrm{CoMnSb}$ for the experimental lattice parameter and the ones compressed and expanded by $2 \%$. First one sees, that upon compression the Fermi level moves in the direction of the conduction band, upon expansion towards the valence band. In both cases, however, the half-metallic character is conserved. To explain this behavior, we first note, that the Fermi level is determined by the metallic DOS in the majority band. As we believe, the shift of $E_{F}$ is determined from the behavior of the $\mathrm{Sb} p$-states, in particular by the large extension of these states as compared to the $d$ states. Upon compression the $p$-states are squeezed and hybridize stronger, thus pushing the $d$-states and the Fermi level to higher energies, i.e. towards the minority conduction band. In addition the $\mathrm{Mn} d$ and $\mathrm{Ni}$ or Co $d$ states hybridize stronger, which increases the size of the gap. Upon expansion the opposite effects are observed. In the case of $\mathrm{NiMnSb}$ and for the experimental lattice constant the gap-width is $\sim 0.4$ $\mathrm{eV}$. When the lattice is expanded by $2 \%$ the gap shrinks to $0.25 \mathrm{eV}$ and when compressed by $2 \%$ the gap-width is increased by $0.1 \mathrm{eV}$ with respect to the experimental lattice constant and is now $0.5 \mathrm{eV}$. Similarly in the case of $\mathrm{CoMnSb}$, the gap is $0.8 \mathrm{eV}$ for the experimental lattice constant, 0.65 for the $2 \%$ expansion and $0.9 \mathrm{eV}$ for the case of the $2 \%$ compression.

For the full-Heusler alloys the pressure dependence has been recently studied by Picozzi et al. 82 for $\mathrm{Co}_{2} \mathrm{MnSi}, \mathrm{Co}_{2} \mathrm{MnGe}$ and $\mathrm{Co}_{2} \mathrm{MnSn}$, using both the LDA and the somewhat more accurate GGA. The general trends are similar: the minority gap increases with compression, and the Fermi level moves in the direction of the conduction band. For example in the case of $\mathrm{Co}_{2} \mathrm{MnSi}$ the gap-width is $0.81 \mathrm{eV}$ for the theoretical equilibrium lattice con-

stant of $10.565 \AA$. When the lattice constant is compressed to $\sim 10.15 \AA$, the gap-width increases to about $1 \mathrm{eV}$.

The calculations show that for the considered changes of the lattice constants of $\pm 2 \%$, half-metallicity is preserved. There can be sizeable changes of the local moments, but the total moment remains constants, since $E_{F}$ stays in the gap.

\section{Quaternary Heusler alloys}

We proceed our study by examining the behavior of the so-called quaternary Heusler alloys. 12, 85. In the latter compounds, one of the four sites is oc- 

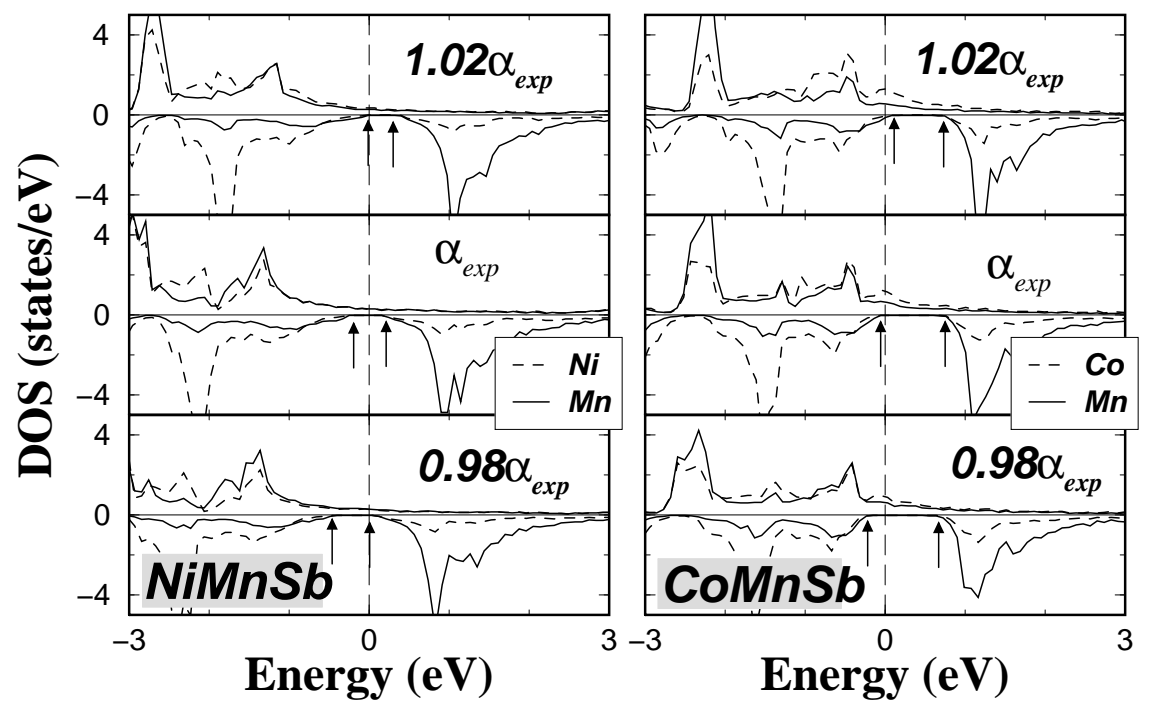

Fig. 12. Atom-resolved DOS for the experimental lattice parameter for NiMnSb and $\mathrm{CoMnSb}$, compared with the once compressed or expanded by $2 \%$. With the small arrow we denote the edges of the minority gap.

cupied by two different kinds of neighboring elements like $\mathrm{Co}_{2}\left[\mathrm{Cr}_{1-x} \mathrm{Mn}_{x}\right] \mathrm{Al}$ where the $\mathrm{Y}$ site is occupied by $\mathrm{Cr}$ or Mn atoms To perform this study we used the KKR method within the coherent potential approximation (CPA) as implemented by H. Akai 23], which has been already used with success to study the magnetic semiconductors 23. For all calculations we assumed that the lattice constant varies linearly with the concentration $x$ which has been verified for several quaternary alloys [5, 6]. To our knowledge from the systems under study only $\mathrm{Co}_{2} \mathrm{Cr}_{0.6} \mathrm{Fe}_{0.4} \mathrm{Al}$ has been studied experimentally 83, 84.

We calculated the total spin moment for several quaternary alloys taking into account several possible combinations of chemical elements and assuming in all cases a concentration increment of 0.1 . We resume our results in figure 13. The first possible case is when we have two different low-valent transition metal atoms at the $\mathrm{Y}$ site like $\mathrm{Co}_{2}\left[\mathrm{Cr}_{1-x} \mathrm{Mn}_{x}\right] \mathrm{Al}$. The total spin moment varies linearly between the $3 \mu_{B}$ of $\mathrm{Co}_{2} \mathrm{CrAl}$ and the $4 \mu_{B}$ of $\mathrm{Co}_{2} \mathrm{MnAl}$. In the case of the $\mathrm{Co}_{2}\left[\mathrm{Cr}_{1-x} \mathrm{Fe}_{x}\right] \mathrm{Al}$ and $\mathrm{Co}_{2}\left[\mathrm{Mn}_{1-x} \mathrm{Fe}_{x}\right] \mathrm{Al}$ compounds and up to around $x=0.6$ the total spin moment shows the SP behavior but for larger concentrations it slightly deviates to account for the non-integer moment value of $\mathrm{Co}_{2} \mathrm{FeAl}$ (see figure 12). This behavior is clearly seen in figure 13 when we compare the lines for the $\mathrm{Co}_{2}\left[\mathrm{Mn}_{1-x} \mathrm{Fe}_{x}\right] \mathrm{Al}$ and $\mathrm{Co}_{2} \mathrm{Mn}\left[\mathrm{Al}_{1-x} \mathrm{Sn}_{x}\right]$ compounds; the latter family follow the SP behavior. The second case is when one mixes the $s p$ elements, but as we just mentioned these compounds 


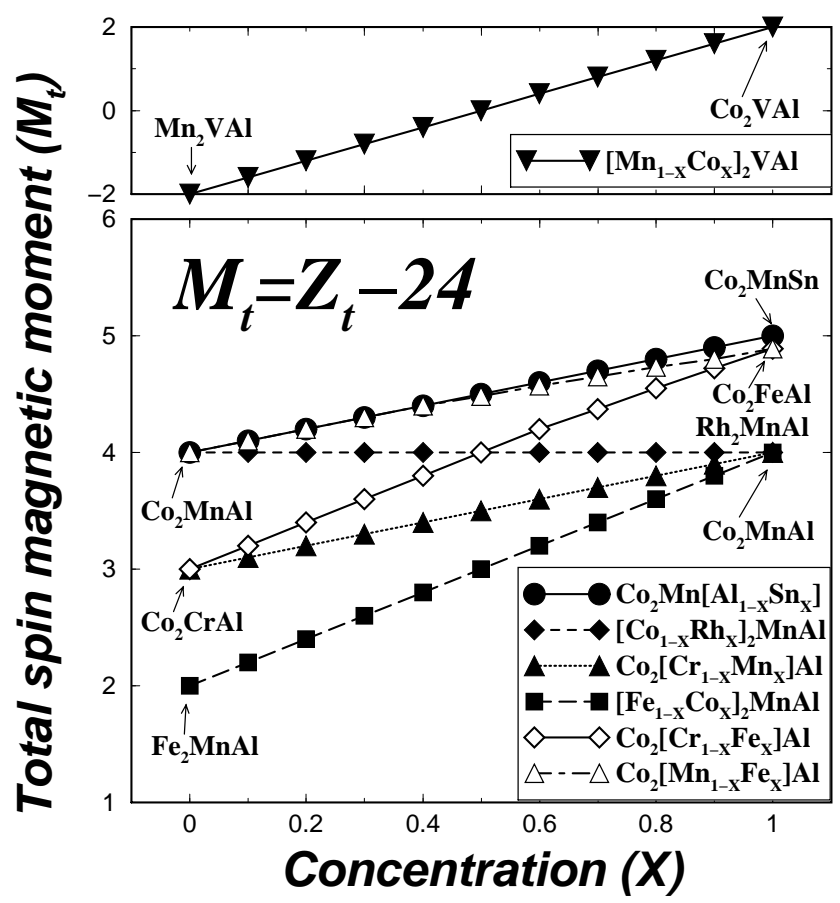

Fig. 13. Calculated total spin moment $M_{t}$ in $\mu_{B}$ for a variety of compounds as a function of the concentration $x(x=0,0.1,0.2, \ldots, 0.9,1)$. We assumed that the lattice constant varies linearly with the concentration $x$. With solid lines the cases obeying the rule $M_{t}=Z_{t}-24$ are shown where $Z_{t}$ and $M_{t}$ are the average total number of valence electrons and the average total moment.

also obey the rule for the total spin moments. The third and final case is to mix the higher valent transition metal atoms like in $\left[\mathrm{Fe}_{1-x} \mathrm{Co}_{x}\right]_{2} \mathrm{MnAl}$ and $\left[\mathrm{Rh}_{1-x} \mathrm{Co}_{x}\right]_{2} \mathrm{MnAl}$ alloys. In the first case the total spin moment varies linearly between the 2 and $4 \mu_{B}$ of $\mathrm{Fe}_{2} \mathrm{MnAl}$ and $\mathrm{Co}_{2} \mathrm{MnAl}$ compounds, respectively. $\mathrm{Rh}$ is isoelectronic to $\mathrm{Co}$ and for the second family of compounds we find a constant integer value of $4 \mu_{B}$ for all the concentrations. A special case is $\mathrm{Mn}_{2} \mathrm{VAl}$ which has less than 24 electrons and the total spin moment is $-2 \mu_{B}$. If now we mix $\mathrm{Mn}$ and $\mathrm{Co}$, we get a family of compounds where the total spin moment varies linearly between the $-2 \mu_{B}$ and the $2 \mu_{B}$ and for $x=0.5$ we get the case of a paramagnetic compound consisting of magnetic elements. Thus all the compounds obey the rule $M_{t}=Z_{t}-24$, showing the Slater-Pauling behavior regardless of the origin of the extra charge.

As a rule of thumb we expect, that for two half-metallic alloys like $X Y Z$ and $X^{\prime} Y Z$ (or $X Y^{\prime} Z, X Y Z^{\prime}$ ), which both lay on the Slater-Pauling curve, also the mixtures like $X_{1-x} X_{x}^{\prime} Y Z$ lay on the Slater Pauling curve, with an average moment of $\left\langle M_{t}\right\rangle=(1-x) M_{t}^{X Y Z}+x M_{t}^{X^{\prime} Y Z}$. However, if these 
intermediate structures are stable, is not guaranteed in particular if the parent compounds are not neighbors on the Slater-Pauling curve..

\section{Point Defects in Half-Metals}

First we would like to discuss some simple rules for point defects in halfmetals. An important problem is, how and by which states the additional or missing charge of the impurity or point defect is screened. There are several mechanisms:

1. Either the point defects is screened metallically by the majority states such that the number of the minority states does not change. If $\Delta Z$ is the valence difference of the impurity, then the total change $\Delta M_{t}$ of the alloy moment is given by $\Delta M_{t}=\Delta Z_{t}$.

2. or the point defect is screened by minority states. These can either be additional states in the gap, which are introduced by the impurity and which, when occupied, lead to $\Delta M_{t}=-\Delta Z_{t}$, or these can be localized states split-off from the minority band which lead to $\Delta M_{t}=+\Delta Z_{t}$, if these states are unoccupied.

3. or both effects occur simultaneously, which is not expected for simple defects.

Note that an isolated point defect cannot change the band gap nor the Fermi level, since these are bulk properties. Also the number of minority states cannot be changed, except when the defect introduces additional resonances in the minority band, or takes out weight from this band by splittingoff states into the gap. As a result, in the dilute limit the band gap and half-metallicity is preserved, but localized states in the gap, either occupied or empty ones, can occur. An exception occurs if a multifold degenerate gapstate is partially occupied and thus fixed at the Fermi level. Then a symmetry lowering Jahn-Teller splitting of the level is expected to occur.

For finite concentrations, impurity states in the gap overlap and fast broaden to form impurity bands. If the impurities are randomly distributed, one can show by applying the coherent potential approximation (CPA) [23], that the band width scales as $\sqrt{c}$, where $c$ is the impurity concentration. For instance, this means, that the bandwidth is for a concentration of $1 \%$ only a factor 3 smaller than for $10 \%$. Therefore the impurity bands broaden very fast with concentration and can soon fill up the band gap, in particular, if the band gap is small and the impurity states are rather extended. Therefore the control of defects and disorder is an important problem for the application of Heusler alloys in spin electronics.

Unfortunately there are very few theoretical investigations for defects in half-metallic Heusler alloys. Picozzi and coworkers have recently studies antisite defects in $\mathrm{Co}_{2} \mathrm{MnGe}$ and $\mathrm{Co}_{2} \mathrm{MnSi}$ [86. For details we refer to their 
review in this volume. Here we address only two screening aspects. A Mnantisite atom on the Co position represents an impurity with $\Delta Z_{t}=-2$. It can either be screened by minority states by pulling two states out-off the minority valence band in the energy region above $E_{F}$. However, this is very difficult, since the minority states are basically Co states with small Mn admixture. Therefore the Mn antisite is screened by shifting $2 \mathrm{Mn}$-states out-off the occupied majority band above the Fermi level, thus decreasing the total moment, such that $\Delta M_{t}=-2$. A Co antisite atom on a Mn position is another interesting case with $\Delta Z_{t}=+2$. Since the majority band is nearly filled and a further filling would lead to an increase of the moment, which a Co atom cannot sustain, the additional charge is provided by the minority states by pulling a double degenerate $e_{u}$-state from the empty Co $e_{u}$-band in the energy region slightly below $E_{F}$, thus decreasing the moment by $-2 \mu_{B}$.

Orgassa and coworkers [44] have investigated the effect of disorder on the electronic structure of NiMnSb. In particular they discuss the effect of impurity gap states on the minority DOS and how the impurity bands broaden and fill the gap for higher concentrations of antisites. While the Mn antisite on $\mathrm{Ni}$ position is screened by majority states, not leading to a state in the gap, the $\mathrm{Ni}$ antisite on the Mn position introduces a (three-fold degenerate) $d$-level below the Fermi level, which broadens into an impurity band with increasing concentration. Already for $1 \%$ of antisite pairs this band comes close to $E_{F}$ and at $5 \%$ half-metallicity disappears and the spin polarization at $E_{F}$ decreases from $100 \%$ to $52 \%$. Thus the general impression is that already a disorder concentration of $1 \%$ is dangerous for the gap and that point defects represent a serious problem for half-metallicity. More theoretical studies are needed.

\section{Effect of Spin-orbit Coupling}

As discussed above in a real crystal defects will destroy the half-metallic band gap since they destroy the perfect crystal periodicity and thus the covalent hybridization leading to the gap. Moreover at finite temperature thermally activated spin-flip scattering, e.g. spin waves, will also induce states within the gap [87, 88. But even in an ideally prepared single crystal at zero temperature, the spin-orbit coupling will introduce states in the half-metallic gap of the minority states (for the spin-down electrons), which are produced by spin-flip scattering of the majority states (with spin-up direction). The KKR calculations presented up to this point were obtained using the scalarrelativistic approximation, thus all-relativistic effects have been taken into account with the exception of the spin-orbit coupling. To study the latter effect the KKR method has been extended to a fully relativistic treatment by solving the Dirac equation for the cell-centered potentials [69. Thus the spin-orbit coupling, which is a relativistic effect, is automatically taken into account. For more details we refer to reference [89]. 
Although in our method the Dirac equation is solved, it is easier to understand the spin-orbit effect within perturbation theory using the Schrödinger equation. In this framework, we remind that the spin-orbit coupling of the two spin channels is related to the unperturbed potential $V(r)$ around each atom via the angular momentum operator $\boldsymbol{L}$ and the Pauli spin matrix $\boldsymbol{\sigma}$ :

$$
V_{\mathrm{so}}(r)=\frac{1}{2 m^{2} c^{2}} \frac{\hbar}{2} \frac{1}{r} \frac{d V}{d r} \boldsymbol{L} \cdot \boldsymbol{\sigma}=\left(\begin{array}{ll}
V_{\mathrm{so}}^{\uparrow \uparrow} & V_{\mathrm{so}}^{\uparrow \downarrow} \\
V_{\mathrm{So}}^{\perp \uparrow} & V_{\mathrm{so}}^{\downarrow \downarrow}
\end{array}\right)
$$

The $2 \times 2$ matrix form is understood in spinor basis. With $\uparrow$ and $\downarrow$, the two spin directions are denoted. The unperturbed crystal Hamiltonian eigenvalues for the two spin directions are $E_{n \boldsymbol{k}}^{0 \uparrow}$ and $E_{n \boldsymbol{k}}^{0 \downarrow}$, and the unperturbed Bloch eigenfunctions as $\Psi_{n \boldsymbol{k}}^{0 \uparrow}$ and $\Psi_{n \boldsymbol{k}}^{0 \downarrow}$. Then, noting that within the energy range of the spin-down gap there exist no unperturbed solutions $\Psi_{n \boldsymbol{k}}^{0 \downarrow}$ and $E_{n \boldsymbol{k}}^{0 \downarrow}$, the first order solution of the Schrödinger equation for the perturbed wavefunction $\Psi_{n \boldsymbol{k}}^{\downarrow}$ reads for states in the gap:

$$
\Psi_{n \boldsymbol{k}}^{(1) \downarrow}(\boldsymbol{r})=\sum_{n^{\prime}} \frac{\left\langle\Psi_{n^{\prime} \boldsymbol{k}}^{0 \downarrow}\left|V_{\mathrm{SO}}^{\downarrow \uparrow}\right| \Psi_{n \boldsymbol{k}}^{0 \uparrow}\right\rangle}{E_{n \boldsymbol{k}}^{0 \uparrow}-E_{n^{\prime} \boldsymbol{k}}^{0 \downarrow}} \Psi_{n^{\prime} \boldsymbol{k}}^{0 \downarrow}(\boldsymbol{r})
$$

Here, the summation runs only over the band index $n^{\prime}$ and not over the Bloch vectors $\boldsymbol{k}^{\prime}$, because Bloch functions with $\boldsymbol{k}^{\prime} \neq \boldsymbol{k}$ are mutually orthogonal. Close to the crossing point $E_{n \boldsymbol{k}}^{0 \uparrow}=E_{n^{\prime} \boldsymbol{k}}^{0 \downarrow}$ the denominator becomes small and the bands strongly couple. Then one should also consider higher orders in the perturbation expansion. Since at the gap edges there exist spin-down bands of the unperturbed Hamiltonian, this effect can become important near the gap edges. Apart from that, the important result is that in the gap region the spin-down spectral intensity is a weak image of the spin-up one, determined by the majority states $E_{n k}^{0 \uparrow}$ in the energy region of the gap. Since the spin-down DOS is related to $\left|\Psi_{n k}^{(1) \downarrow}\right|^{2}$, it is expected that within the gap the DOS has a quadratic dependence on the spin-orbit coupling strength: $n_{\downarrow}(E) \sim\left(V_{\text {so }}^{\downarrow \uparrow}\right)^{2}$.

Table 3. Calculated spin polarization at the Fermi level $\left[P\left(E_{F}\right)\right]$ and in the middle of the spin-down gap $\left[P\left(E_{M}\right)\right]$, for various Heusler alloys. The alloys PdMnSb and PtMnSb present a spin-down gap, but are not half-metallic, as $E_{F}$ is slightly below the gap.

\begin{tabular}{rrrrrr}
\hline Compound FeMnSb CoMnSb NiMnSb PdMnSb PtMnSb \\
\hline$P\left(E_{F}\right)$ & $99.3 \%$ & $99.0 \%$ & $99.3 \%$ & $40.0 \%$ & $66.5 \%$ \\
$P\left(E_{M}\right)$ & $99.4 \%$ & $99.5 \%$ & $99.3 \%$ & $98.5 \%$ & $94.5 \%$ \\
\hline
\end{tabular}

In table 3 we present the results for several Heusler alloys. In addition to NiMnSb, the cases of FeMnSb, CoMnSb, PdMnSb, and PtMnSb have been 
studied. Although the last two are not half-metallic (a spin-down gap exists, but $E_{F}$ enters slightly into the valence band), it is instructive to examine the DOS in the gap region and see how the spinpolarization decreases as one changes to heavier elements $(\mathrm{Ni} \rightarrow \mathrm{Pd} \rightarrow \mathrm{Pt})$. Here the spinpolarization is $P(E)$ is defined by the ratio

$$
P(E)=\frac{n^{\uparrow}(E)-n^{\downarrow}(E)}{n^{\uparrow}(E)+n^{\downarrow}(E)}
$$

For the Heusler alloys CoMnSb, FeMnSb, NiMnSb, PdMnSb, and PtMnSb, two quantities are shown: the spin polarization at $E_{F}, P\left(E_{F}\right)$, and in the middle of the gap, $P\left(E_{M}\right)$. The last quantity reflects the strength of the spin-orbit induced spin flip scattering, while the first is relevant to our considerations only when $E_{F}$ is well within the gap (which is not the case for $\mathrm{PdMnSb}$ and PtMnSb). Clearly, the compounds including 3d transition elements (NiMnSb, CoMnSb, and FeMnSb) show high spin polarization with small variation as we move along the $3 \mathrm{~d}$ row of the periodic table from $\mathrm{Ni}$ to $\mathrm{Fe}$. In contrast, when we substitute Ni with its isoelectronic $\mathrm{Pd}$ the number of the induced minority states at the middle of the gap increases and $P\left(E_{M}\right)$ drops drastically, and much more when we change Pd for the isoelectronic $\mathrm{Pt}$. This trend is expected, since it is known that heavier elements are characterized by stronger spin-orbit coupling. But in all cases the alloys retain a region of very high spin-polarization, instead of a real gap present in the scalar relativistic calculations, and thus this phenomenon will not be important for realistic applications. Defects, thermally activated spin-flip scattering, surface or interfaces states will have a more important effect on the spin-polarization.

Since we can include the spin-orbit coupling to the calculations, we are also able to determine the orbital moments of the alloys (see reference 90] for more details). The minority valence bands are completely occupied while for the majority ones the density of states at the Fermi level is usually very small since most of the $d$ states are occupied. Thus the orbital moments are almost completely quenched and their absolute values are negligible with respect to the spin magnetic moments. Also Picozzi and collaborators have studied the orbital magnetism of $\mathrm{Co}_{2} \mathrm{Mn}-\mathrm{Si}$, -Ge and -Sn compounds and have reached to similar conclusions [82. This also means that the magnetic anisotropy of the half-metallic Heusler alloys is expected to be rather small.

\section{Surface Properties}

Surfaces can change the bulk properties severely, since the coordination of the surface atoms is strongly reduced. Based on the experiences from ferromagnets and semiconductors, two effects should be particularly relevant for the surfaces of half-metals: (i) for ferromagnets the moments of the surface atoms are strongly enhanced due to the missing hybridization with the cutoff neighbors, and (ii) for semiconductors surface states appear in the gap, 
such that the surface often becomes metallic. Also this is a consequence of the reduced hybridization, leading to dangling bond states in the gap.

In this section we review some recent calculations for the (001) surfaces of half- and full-Heusler alloys. We will not touch the more complex problem of the interfaces with semiconductors, since this is treated in the review chapter of Picozzi et al. in this book. As a model we firstly consider the (001)surface of NiMnSb. Jenkins and King have investigated the MnSb-terminated $\operatorname{NiMnSb}(001)$ surface in detail and have shown that the surface relaxations are very small; $\mathrm{Sb}$ atoms relax outwards while $\mathrm{Mn}$ atoms relax inwards with a total buckling of $0.06 \AA$ [4]. Therefore we neglect the relaxations and assume an "ideal" epitaxy. The second possible termination, with a Ni-atom and an unoccupied site at the surface, is not considered since the configuration is likely to be unstable or to show large relaxations of the Ni-surface atoms.

As mentioned above, in the case of the MnSb-terminated (001) NiMnSb surface, Jenkins and King have shown that there are two surface states 48]. The lower lying state (at $0.20 \mathrm{eV}$ above the $\bar{\Gamma}$ point) is due to the interaction between $e_{g}$-like dangling bond states located at the Mn atoms. The second surface state, which is also higher in energy $(0.44 \mathrm{eV}$ above the $\bar{\Gamma}$ point $)$ rises from the hybridization between $t_{2 g}$-like orbitals of Mn with $p$-type orbitals of $\mathrm{Sb}$. The first surface state disperses downwards along the [110] direction while the second surface state disperses upwards along the same direction. Their behavior is inversed along the [110] direction. The two surface states cross along the $[1 \overline{1} 0]$ direction bridging the minority gap between the valence and the conduction band. Along the other directions anticrossing occurs leading to band-gaps. Of interest are also the saddle-like structures around the zone center which manifest as van Hove singularities in the DOS.

In figure 14] we present the atom- and spin-projected density of states (DOS) for the $\mathrm{Mn}$ and $\mathrm{Sb}$ atoms in the surface layer and the $\mathrm{Ni}$ and vacant site in the subsurface layer for the MnSb terminated $\mathrm{NiMnSb}(001)$ surface. We compare the surface DOS with the bulk calculations (dashed line). With the exception of the gap region, the surface DOS is very similar to the bulk case. The Ni atom in the subsurface layer presents practically a half-metallic behavior with an almost zero spin-down DOS, while for the bulk there is an absolute gap. The spin-down band of the vacant site also presents a very small DOS around the Fermi level. The Mn and Sb atoms in the surface layer show more pronounced differences with respect to the bulk, and within the gap there is a very small Mn- $d$ and Sb- $p$ DOS. These intensities are due to the two surface states found by Jenkins and King [48. These two surface states are strongly localized at the surface layer as at the subsurface layer there is practically no states inside the gap. Our results are in agreement with the experiments of Ristoiu et al. [40] who in the case of a MnSb well ordered (001) surface measured a high spin-polarization. Finally we should mention that the spin moment of the Mn atom at the surface is increased by $0.3 \mu_{B}$ 
Surface Layer

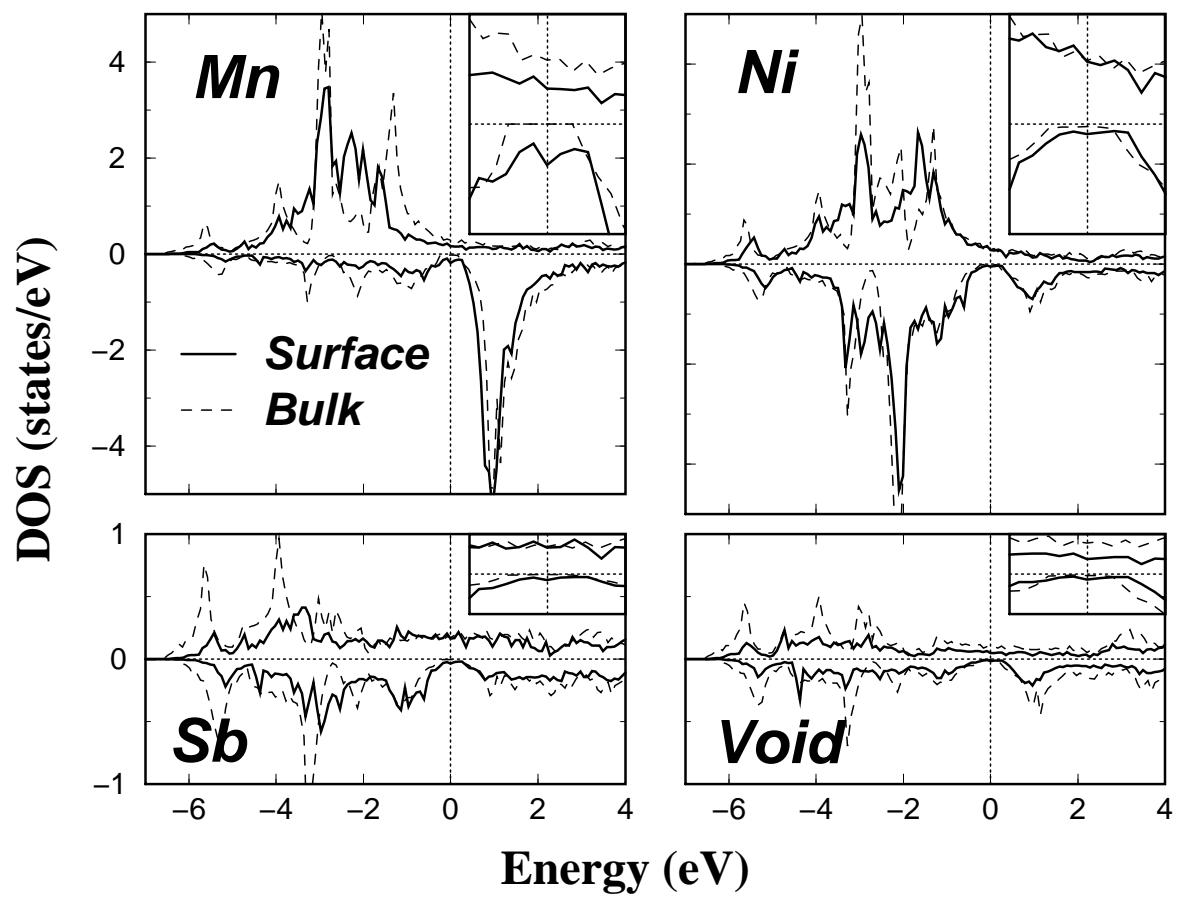

Fig. 14. Spin- and atom-projected DOS for the MnSb-terminated NiMnSb(001) surface. In the insets we have blown up the region around the gap (between -0.5 and $0.5 \mathrm{eV}$ ). The dashed lines give the local DOS of the atoms in the bulk.

with respect to the bulk NiMnSb and reaches the $\sim 4 \mu_{B}$ due to the missing hybridization with the cut-off Ni neighbors.

It is also interesting to examine the spin-polarization at the Fermi level. In table 4 we have gathered the number of spin-up and spin-down states at the Fermi level for each atom at the surface and the subsurface layer for the MnSb-terminated surfaces for different compounds. We calculated the spinpolarization as the ratio between the number of spin-up states minus the number of spin-down states over the total DOS at the Fermi level. $P_{1}$ corresponds to the spin-polarization when we take into account only the surface layer and $P_{2}$ if we sum the DOS of the surface and subsurface layers. $P_{2}$ simulates reasonably well the experimental situation as the spin-polarization in the case of films is usually measured by inverse photoemission which probes only the surface of the sample 91]. In all cases the inclusion of the subsurface layer increased the spin-polarization since naturally the second layer is expected to be more bulk-like. In the case of the Ni terminated surface, the spin-up DOS at the Fermi level is equal to the spin-down DOS and the net 
local polarization $P_{2}$ is zero. In the case of the MnSb terminated surface the spin-polarization increases and now $P_{2}$ reaches a value of $38 \%$, which means that the spin-up DOS at the Fermi level is about two times the spin-down DOS. The main difference between the two different terminations is the contribution of the $\mathrm{Ni}$ spin-down states. In the case of the MnSb surface the $\mathrm{Ni}$ in the subsurface layer has a spin-down DOS at the Fermi level of 0.05 states/eV, while in the case of the Ni-terminated surface the Ni spin-down DOS at the Fermi level is 0.40 states/eV decreasing considerably the spinpolarization for the Ni terminated surface; the Ni spin-up DOS is the same for both terminations. It is interesting also to note that for both surfaces the net $\mathrm{Mn}$ spin-polarization is close to zero while $\mathrm{Sb}$ atoms in both cases show a large spin-polarization and the number of the Sb spin-up states is similar to the number of Mn spin-up states, so that $\mathrm{Sb}$ and not $\mathrm{Mn}$ is responsible for the large spin-polarization of the MnSb layer in both surface terminations. The calculated $P_{2}$ value of $38 \%$ for the MnSb terminated surface is smaller than the experimental value of $67 \%$ obtained by Ristoiu and collaborators 40] for a thin-film terminated in a MnSb stoichiometric alloy surface layer. But experimentally no exact details of the structure of the film are known and the comparison between experiment and theory is not straightforward.

Table 4. Atomic-resolved spin-up and spin-down local DOS at the Fermi level in states/eV units. Polarization ratios at the Fermi level are calculated taking into account only the surface layer $P_{1}$, and the sum of the surface and subsurface layers $P_{2}$.

\begin{tabular}{|c|c|c|c|c|c|c|}
\hline & \multicolumn{4}{|c|}{ MnSb (MnGe or CrAl) -termination } & \multirow[b]{2}{*}{$P_{1}\left(\frac{\uparrow-\downarrow}{\uparrow+\downarrow}\right)$} & \multirow{2}{*}{$P_{2}\left(\frac{\uparrow-\downarrow}{\uparrow+\downarrow}\right)$} \\
\hline & $\begin{array}{r}\text { Surface } \\
\operatorname{Mn}(\uparrow / \downarrow)\end{array}$ & $\begin{array}{l}\text { Layer } \\
\mid \mathrm{Sb}(\uparrow / \downarrow)\end{array}$ & $\begin{array}{r}\text { Subsurface } \\
\mathrm{Ni}[\mathrm{Co}, \mathrm{Pt}](\uparrow / \downarrow)\end{array}$ & $\begin{array}{l}\text { Layer } \\
\mid \text { Void }(\uparrow / \downarrow)\end{array}$ & & \\
\hline $\mathrm{Ni}$ & $0.16 / 0.19$ & $0.17 / 0.03$ & $0.28 / 0.05$ & $0.05 / 0.02$ & $26 \%$ & $38 \%$ \\
\hline $\mathrm{CoM}$ & $0.23 / 0.27$ & $0.16 / 0.07$ & $0.91 / 0.15$ & $0.0^{7}$ & $6 \%$ & $46 \%$ \\
\hline $\mathrm{PtMnSb}$ & $0.21 / 0.24$ & $0.31 / 0.06$ & $0.38 / 0.04$ & $0.08 / 0.02$ & $26 \%$ & $46 \%$ \\
\hline
\end{tabular}

In the case of the full-Heusler alloys containing Mn the results are similar to the ones obtained for the NiMnSb compound. For the MnGe-terminated (001) surface the induced Mn minority surface states locally completely kill the spin polarization. This is clearly seen in the bottom panel of figure 15 where we have plotted the DOS around the Fermi level for the Mn and Ge atoms at the surface layer and the Co atoms at the subsurface layer. Note that also for Co at the subsurface the local net spin polarization is zero. But in the case of $\mathrm{Co}_{2} \mathrm{CrAl}$ results differ considerably. In line with the reduction of the total valence electrons by 2 , the $\mathrm{Cr}$ moment is rather small $\left(1.54 \mu_{B}\right)$ yielding a total moment of only $3 \mu_{B}$ instead of $5 \mu_{B}$ for $\mathrm{Co}_{2} \mathrm{MnGe}$. The Co terminated $\mathrm{Co}_{2} \mathrm{CrAl}(001)$ surface shows a similar behavior as the corresponding surface of $\mathrm{Co}_{2} \mathrm{MnGe}$, being in both cases dominated by a strong Co peak in the gap 


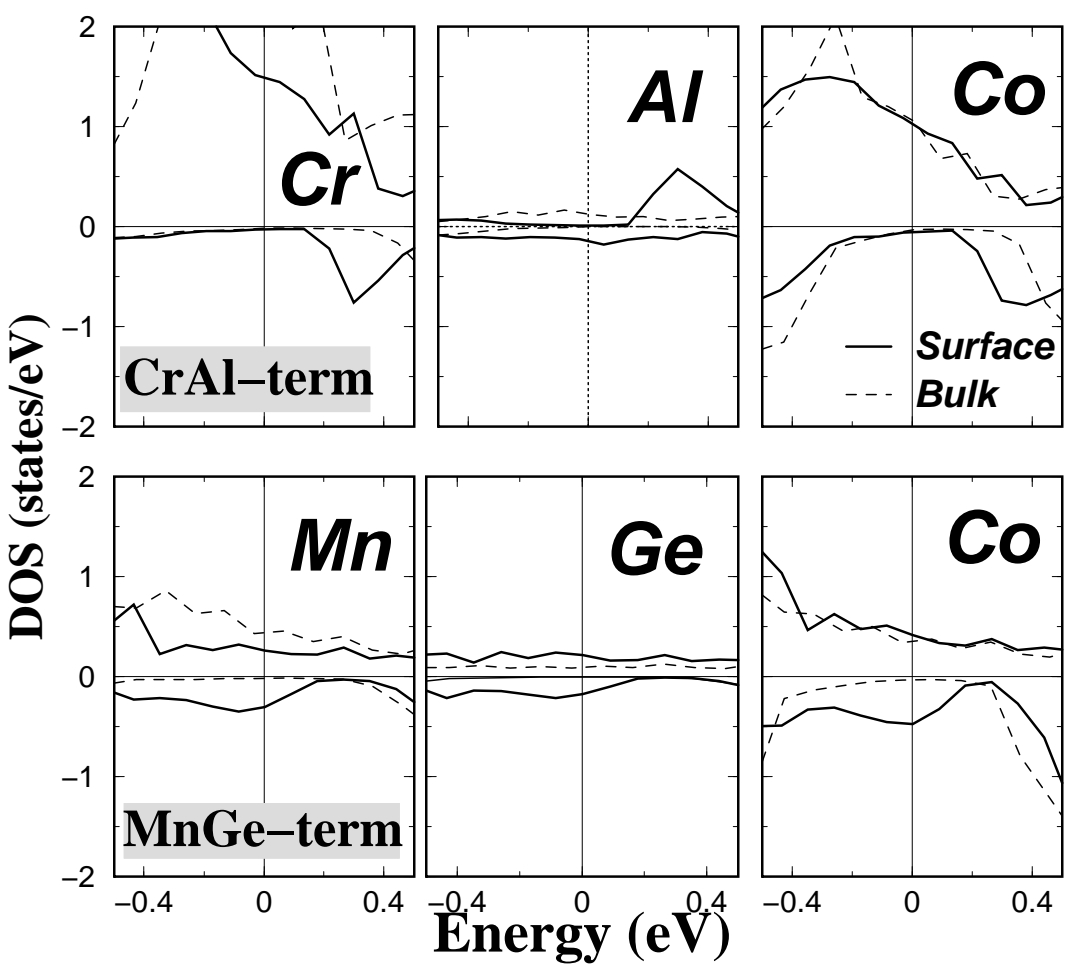

Fig. 15. Atom- and spin-projected DOS for the MnGe and CrAl terminated (001) surfaces of $\mathrm{Co}_{2} \mathrm{MnGe}$ and $\mathrm{Co}_{2} \mathrm{CrAl}$, respectively. With dashed line: the bulk results.

region of the minority band. However the $\mathrm{CrAl}$ terminated $\mathrm{Co}_{2} \mathrm{CrAl}$ surface behaves very differently, being driven by the large surface enhancement of the Cr moment from $1.54 \mu_{B}$ to $3.12 \mu_{B}$. As a consequence the splitting of the $\mathrm{Cr}$ peaks in the majority and minority bands is even enlarged and in particular in the minority band the pseudogap is preserved. Thus this surface is a rare case, since for all the other surfaces studied in this paper, the half-metallicity is destroyed by surface states. If we look closer at the gap region (see figure 15D) for the $\mathrm{CrAl}$ surface we find that the Al DOS still has some weight in the gap region. Thus compared to NiMnSb there is still one surface state left, which has only an $\mathrm{Al} p$-component, but no $d$-admixture from the $\mathrm{Cr}$ atom. However in total the surface keeps a high degree of spin polarization: i.e. $P_{2}$ is $84 \%$.

The increase of the spin magnetic moment of the surface atoms due to their lower coordination with respect to the bulk can be better understood in the case of the transition-metal pnictides and chalcogenides which crystallize in the zinc-blende structure. Using $\mathrm{CrAs}$ and $\mathrm{CrSe}$ as examples, Galanakis and Mavropoulos have shown that the Cr-terminated (001) surfaces of these 
alloys retain the half-metallicity of the bulk compounds [17. Contrary to the $\mathrm{CrAl}$-terminated $\mathrm{Co}_{2} \mathrm{CrAl}(001)$ there is no $s p$ atom at the surface layer now to induce a surface state. Thus the situation is simpler to understand as compared to the Heusler alloys. In the bulk case, Cr has four As or Se atoms as first neighbors. Thus the bonding can be described in terms of four directional bonds around each $\mathrm{Cr}$ or $\mathrm{As}(\mathrm{Se})$ atom, similar to the case of binary semiconductors where $s p^{3}$ hybrids are being formed. Each $\mathrm{Cr}$ atom provides $0.75 e^{-}$per Cr-As bond and $0.5 e^{-}$per Cr-Se bond. The $\mathrm{Cr}$ atom at the surface looses two out of its four As or Se first neighbors and thus regains 1.5 electrons in the case of $\mathrm{CrAs}(001)$ and 1 electron in the case of $\mathrm{CrSe}(001)$. The extra electrons now fill up only spin up states and thus the total spin moment at the surface (adding the spin moments of the Cr surface atom and of the As or Se at the subsurface layer) is enhanced by exactly 1.5 $\mu_{B}$ and $1 \mu_{B}$ with respect to the bulk for CrAs and CrSe, respectively [17. This rule for the total spin moment can be also generalized to the case of the interfaces with semiconductors with the condition that half-metallicity is preserved [17].

\section{Summary and Outlook}

In this review we have given an introduction into the electronic structure and the resulting magnetic properties of half-metallic Heusler alloys, which represent interesting hybrids between metallic ferromagnets and semiconductors. Many unusual features arise from the half-metallicity induced by the gap in the minority band, and therefore the understanding of the gap is of central importance.

For the half-Heusler alloys like NiMnSb, crystallizing in the $C 1_{b}$ structure, the gap arises from the hybridization between the $d$-wavefunctions of the lower-valent transition metal atom (e.g. Mn) with the $d$-wavefunctions of the higher-valent transition metal atom (e.g. Ni). Thus the $d-d$ hybridization leads to 5 occupied bonding bands, which have a larger $\mathrm{Ni}$ and smaller $\mathrm{Mn}$ admixture. These states form the valence band, being separated by a band gap from the conduction band which is formed by five antibonding hybrids with a large Mn $d$ - and a small Ni $d$-admixture. The role of the $s p$ atoms like $\mathrm{Sb}$ is very different. Firstly they are important for the bonding, in particular for the stabilization of the $C 1_{b}$ structure. Secondly the $s p$ atom creates for each spin direction one $s$ and three $p$ bands in the energy region below the $d$ states which by hybridization can accommodate also transition metal electrons, such that e.g. Sb formally acts like a $\mathrm{Sb}^{-3}$ and $\mathrm{Sn}$ as a $\mathrm{Sn}^{-4}$ anion. In this way the effective number of valence $d$-electrons can be changed by the valence of the $s p$ elements.

Since the minority valence band consist of 9 bands, compounds with 18 valence electrons like CoTiSb have the same density of states for both spin directions and are semiconductors. More general, compounds with a total 
number of $Z_{t}$ valence electrons per unit cell are ferromagnets and have an integer total spin moment of $M_{t}=Z_{t}-18$, since $Z_{t}-18$ is the number of uncompensated spins. For instance, NiMnSb has 22 valence electrons and therefore a total moment of exactly $4 \mu_{B}$. This relation is similar to the well known Slater-Pauling behavior observed for binary transition-metal alloys and allows to classify the half-metallic $C 1_{b}$ Heusler alloys into classes with integer moments between 0 and $5 \mu_{B}$. The maximum moment of $5 \mu_{B}$ is difficult to achieve, since it requires that all majority $d$-sates are occupied.

In the case of the full-Heusler alloys like $\mathrm{Co}_{2} \mathrm{MnGe}$, there are, in addition to the Co-Mn bonding and antibonding $d$-hybrids, also Co states which cannot hybridize with both the $\mathrm{Mn}$ and the Ge atoms and are exclusively localized at the two Co sublattices. Thus in addition to the 5 Co-Mn bonding and 5 Co-Mn antibonding bands, there exist 5 such "non-bonding" bands which are only splitted-up by the weaker Co-Co hybridization into 3 occupied $d$ states of $t_{1 u}$ symmetry and 2 unoccupied $e_{u}$ states, which are located just below and just above the Fermi level such that the indirect gap in these materials is smaller than in the half-Heuslers. Due to the additional 3 occupied $t_{1 u}$ cobalt bands, the full-Heusler alloys have 12 occupied minority bands instead of 9 in the case of the half-Heusler compounds and their relation for the total spin magnetic moment becomes $M_{t}=Z_{t}-24$. Thus systems like $\mathrm{Fe}_{2} \mathrm{VAl}$ with 24 valence electrons are semiconductors, $\mathrm{Co}_{2} \mathrm{VAl}$ (26 valence electrons) has a total spin moment of $2 \mu_{B}, \mathrm{Co}_{2} \mathrm{CrAl} 3 \mu_{B}, \mathrm{Co}_{2} \mathrm{MnAl} 4 \mu_{B}$ and finally $\mathrm{Co}_{2} \mathrm{MnSi}$ which has 29 valence electrons has a total spin moment of $5 \mu_{B}$. The maximal total spin moment for these alloys is $7 \mu_{B}$, but as has been shown even the $6 \mu_{B}$ are unlikely to be achieved.

Having understood the basic elements of the electronic structure, there is still a long way to go for understanding the half-metallic behavior of the real materials. Since the existence of the minority gap is central for any application of half-metals in spintronics, it is of great importance to understand and control all mechanisms that can destroy the gap. Firstly we have discussed that spin-orbit interaction couples the two spin-bands and induces states in the gap; however this effect is weak and the spinpolarization remains in most cases as high as $\sim 99 \%$. While this effect exists already in the ground state, there are, secondly, excitation effects leading to states in the gap. In the simplest approach one can consider in the adiabatic approximation "static spin waves", which are superpositions of spin-up and spin-down states. At a finite temperature the spinwaves excitations will then smear out the gap 88. A more ambitious treatment of the interaction with magnons leads to non-quasiparticle excitations in the minority gap above the Fermi level 92 and at the Fermi level the minority density of states strongly depends on the temperature.

Thirdly and most importantly all kind of defects are expected to lead to states in the gap. We have discussed this qualitatively for the case of point defects arising from substitutional disorder and refer to the contribution of 
Picozzi et al. in this volume for realistic calculations. We feel that many more calculations and experiments are needed; the aim is to find systems, which either do not lead to states in the gap (which is presumably not possible) or systems with particularly high defect formation energies or sufficiently low annealing temperatures. Equally important is the control of surface and interface states in the gap, the latter are in particular important for interfaces to semiconductors. Here it should be possible to find junctions which do not have interface states at the Fermi level. The case of $\mathrm{Co}_{2} \mathrm{CrAl}$ discussed in this paper shows, that the occurrence of the transition-metal induced surface state at the Fermi level can be suppressed by the increase of the $\mathrm{Cr}$ spin moment at the surface. Finally we add that from point of view of transport a single interface state does not affect the magnetoconductance since the wavefunction is orthogonal to all bulk states incident to the interface. It is the interaction with other defect states in bulk systems and/or with surface defects which make these states conducting.

\section{References}

1. I. Žutić, J. Fabian, and S. Das Sarma: Rev. Mod. Phys. 76, 323 (2004)

2. S.A. Wolf, D.D. Awschalom, R.A. Buhrman, J.M. Daughton, S. von Molnár, M.L. Roukes, A.Y. Chtchelkanova, and D.M. Treger: Science 294, 1488 (2001); G.A. Prinz: Science 282, 1660 (1998); G.A. Prinz: J. Magn. Magn. Mater. 200, 57 (1999)

3. J. de Boeck, W. van Roy, J. Das, V. Motsnyi, Z. Liu, L. Lagae, H. Boeve, K. Dessein, and G. Borghs: Semicond. Sci. Tech. 17, 342 (2002); J. de Boeck, W. van Roy, V. Motsnyi, Z. Liu, K. Dessein, and G. Borghs: Thin Solid Films 412, 3 (2002)

4. F. Heusler: Verh. Dtsch. Phys. Ges. 5, 219 (1903)

5. P.J. Webster and K.R.A. Ziebeck. In: Alloys and Compounds of d-Elements with Main Group Elements. Part 2., Landolt-Börnstein, New Series, Group III, vol 19c, ed by H.R.J. Wijn, (Springer, Berlin 1988) pp 75-184

6. K.R.A. Ziebeck and K.-U. Neumann. In: Magnetic Properties of Metals, Landolt-Börnstein, New Series, Group III, vol 32/c, ed by H.R.J. Wijn, (Springer, Berlin 2001) pp 64-414

7. J. Pierre, R.V. Skolozdra, J. Tobola, S. Kaprzyk, C. Hordequin, M.A. Kouacou, I. Karla, R. Currat, and E. Lelièvre-Berna: J. Alloys Comp. 262-263, $101(1997)$

8. J. Tobola, J. Pierre, S. Kaprzyk, R.V. Skolozdra, and M.A. Kouacou: J. Phys.: Condens. Matter 10, 1013 (1998); J. Tobola and J. Pierre: J. Alloys Comp. 296, 243 (2000); J. Tobola, S. Kaprzyk, and P. Pecheur: Phys. St. Sol. (b) 236, $531(2003)$

9. R.A. de Groot. F.M. Mueller, P.G. van Engen, and K.H.J. Buschow: Phys. Rev. Lett. 50, 2024 (1983)

10. I. Galanakis, N. Papanikolaou, and P. H. Dederichs: Phys. Rev. B 66, 134428 (2002)

11. I. Galanakis, P. H. Dederichs, and N. Papanikolaou: Phys. Rev. B 66, 174429 (2002) 
12. I. Galanakis: J. Phys.: Condens. Matter 16, 3089 (2004);

13. M. Zhang, X. Dai, H. Hu, G. Liu, Y. Cui, Z. Liu, J. Chen, J. Wang, and G. Wu: J. Phys: Condens. Matter 15, 7891 (2003); M. Zhang, Z. Liu, H. Hu, G. Liu, Y. Cui, G. Wu, E. Brück, F.R. de Boer, and Y. Li : J. Appl. Phys. 95, 7219 (2004).

14. R.J. Soulen Jr., J.M. Byers, M.S. Osofsky, B. Nadgorny, T. Ambrose, S.F. Cheng, P.R. Broussard, C.T. Tanaka, J. Nowak, J.S. Moodera, A. Barry, and J.M.D. Coey: Science 282, 85 (1998)

15. H. Kato, T. Okuda, Y. Okimoto, Y. Tomioka, K. Oikawa, T. Kamiyama, and Y. Tokura: Phys. Rev. B 69, 184412 (2004)

16. T. Shishidou, A.J. Freeman, and R. Asahi: Phys. Rev. B 64, 180401 (2001)

17. I. Galanakis: Phys. Rev. B 66, 012406 (2002); I. Galanakis and Ph. Mavropoulos: Phys. Rev. B 67, 104417 (2003); Ph. Mavropoulos and I. Galanakis: J. Phys.: Condens. Matter 16, 4261 (2004)

18. S. Sanvito and N.A. Hill: Phys. Rev. B 62, 15553 (2000); A. Continenza, S. Picozzi, W.T. Geng, and A.J. Freeman: Phys. Rev. B 64, 085204 (2001); B.G. Liu: Phys. Rev. B 67, 172411 (2003); B. Sanyal, L. Bergqvist, and O. Eriksson: Phys. Rev. B 68, 054417 (2003); W.-H. Xie, B.-G. Liu, and D.G. Pettifor: Phys. Rev. B 68, 134407 (2003); W.-H. Xie, B.-G. Liu, and D.G. Pettifor: Phys. Rev. Lett. 91, 037204 (2003); Y.Q. Xu, B.-G. Liu, and D.G. Pettifor: Phys. Rev. B 68, 184435 (2003); M. Zhang et al.: J. Phys: Condens. Matter 15, 5017 (2003); C.Y. Fong, M.C. Qian, J.E. Pask, L.H. Yang, and S. Dag: Appl. Phys. Lett. 84, 239 (2004); J.E. Pask, L.H. Yang, C.Y. Fong, W.E. Pickett, and S. Dag: Phys. Rev. B 67, 224420 (2003); J.-C. Zheng and J.W. Davenport: Phys. Rev. B 69, 144415 (2004)

19. H. Akinaga, T. Manago, and M. Shirai: Jpn. J. Appl. Phys. 39, L1118 (2000); M. Mizuguchi, H. Akinaga, T. Manago, K. Ono, M. Oshima, and M. Shirai: J. Magn. Magn. Mater. 239, 269 (2002); M. Mizuguchi, H. Akinaga, T. Manago, K. Ono, M. Oshima, M. Shirai, M. Yuri, H.J. Lin, H.H. Hsieh, and C.T. Chen: J. Appl. Phys. 91, 7917 (2002); M. Mizuguchi, M. K. Ono, M. Oshima, J. Okabayashi, H. Akinaga, T. Manago, and M. Shirai: Surf. Rev. Lett. 9, 331 (2002); M. Nagao, M. Shirai, and Y. Miura: J. Appl. Phys. 95, 6518 (2004); K. Ono, J. Okabayashi, M. Mizuguchi, M. Oshima, A. Fujimori, and H. Akinaga: J. Appl. Phys. 91, 8088 (2002); M. Shirai: Physica E 10, 143 (2001); M. Shirai: J. Appl. Phys. 93, 6844 (2003);

20. J.H. Zhao, F. Matsukura, K. Takamura, E. Abe, D. Chiba, and H. Ohno: Appl. Phys. Lett. 79, 2776 (2001); J.H. Zhao, F. Matsukura, K. Takamura, E. Abe, D. Chiba, Y. Ohno, K. Ohtani, and H. Ohno: Mat. Sci. Semicond. Proc. 6, 507 (2003)

21. M. Horne, P. Strange, W.M. Temmerman, Z. Szotek, A. Svane, and H. Winter: J. Phys.: Condens. Matter 16, 5061 (2004)

22. A. Stroppa, S. Picozzi, A. Continenza, and A. J. Freeman: Phys. Rev. B 68, 155203 (2003)

23. H. Akai: Phys. Rev. Lett. 81, 3002 (1998)

24. J.-H. Park, E. Vescovo, H.-J. Kim, C. Kwon, R. Ramesh, and T. Venkatesan: Nature 392, 794 (1998)

25. S. Datta and B. Das: Appl. Phys. Lett. 56, 665 (1990)

26. K.A. Kilian and R.H. Victora: J. Appl. Phys. 87, 7064 (2000)

27. C.T. Tanaka, J. Nowak, and J.S. Moodera: J. Appl. Phys. 86, 6239 (1999) 
28. J.A. Caballero, Y.D. Park, J.R. Childress, J. Bass, W.-C. Chiang, A.C. Reilly, W.P. Pratt Jr., and F. Petroff: J. Vac. Sci. Technol. A 16, 1801 (1998); C. Hordequin, J.P. Nozières, and J. Pierre: J. Magn. Magn. Mater. 183, 225 (1998)

29. M.M. Kirillova, A.A. Makhnev, E.I. Shreder, V.P. Dyakina, and N.B. Gorina: Phys. Stat. Sol. (b) 187, 231 (1995)

30. K.E.H.M. Hanssen and P.E. Mijnarends: Phys. Rev. B 34, 5009 (1986); K.E.H.M. Hanssen, P.E. Mijnarends, L.P.L.M. Rabou, and K.H.J. Buschow: Phys. Rev. B 42, 1533 (1990)

31. W. van Roy, M. Wojcik, E. Jdryka, S. Nadolski, D. Jalabert, B. Brijs, G. Borghs, and J. De Boeck: Appl. Phys. Lett. 83, 4214 (2003); W. van Roy, J. de Boeck, B. Brijs, and G. Borghs: Appl. Phys. Lett. 77, 4190 (2000); J.-P. Schlomka, M. Tolan, and W. Press: Appl. Phys. Lett. 76, 2005 (2000)

32. P. Bach, C. Rüster, C. Gould, C.R. Becker, G. Schmidt, and L.W. Molenkamp: J. Cryst. Growth 251, 323 (2003); P. Bach, A.S. Bader, C. Rüster, C. Gould, C.R. Becker, G. Schmidt, L.W. Molenkamp, W. Weigand, C. Kumpf, E. Umbach, R. Urban, G. Woltersdorf, and B. Heinrich: Appl. Phys. Lett. 83, 521 (2003)

33. J. Giapintzakis, C. Grigorescu, A. Klini, A. Manousaki, V. Zorba, J. Androulakis, Z. Viskadourakis, and C. Fotakis: Appl. Surf. Sci. 197, 421 (2002); J. Giapintzakis, C. Grigorescu, A. Klini, A. Manousaki, V. Zorba, J. Androulakis, Z. Viskadourakis, and C. Fotakis: Appl. Phys. Lett. 80, 2716 (2002); C.E.A. Grigorescu, S.A. Manea, M. Mitrea, O. Monnereau, R. Rotonier, L. Tortet, R. Keschawarz, J. Giapintzakis, A. Klini, V. Zorba, J. Androulakis, and C. Fotakis: Appl. Surf. Sci. 212, 78 (2003); S. Gardelis, J. Androulaki, P. Migiakis, J. Giapintzakis, S.K. Clowes, Y. Bugoslavsky, W.R. Branford, Y. Miyoshi, and L.F. Cohen: J. Appl. Phys. textbf95, 8063 (2004)

34. F.B. Mancoff, B.M. Clemens, E.J. Singley, and D.N. Basov: Phys. Rev. B 60, $R 12565$ (1999)

35. W. Zhu, B. Sinkovic, E. Vescovo, C. Tanaka, and J.S. Moodera: Phys. Rev. B 64, R060403 (2001)

36. G.L. Bona, F. Meier, M. Taborelli, E. Bucher, and P.H. Schmidt: Sol. St. Commun. 56, 391 (1985)

37. S.K. Clowes, Y. Mioyoshi, Y. Bugoslavsky, W.R. Branford, C. Grigorescu, S.A. Manea, O. Monnereau, and L.F. Cohen: Phys. Rev. B 69, 214425 (2004)

38. J.A. Caballero, A.C. Reilly, Y. Hao, J. Bass, W.P. Pratt, F. Petroff, and J.R. Childress: J. Magn. Magn. Mat. 198-199, 55 (1999); R. Kabani, M. Terada, A. Roshko, and J.S. Moodera: J. Appl. Phys. 67, 4898 (1990)

39. C.T. Tanaka, J. Nowak, and J.S. Moodera: J. Appl. Phys. 81, 5515 (1997)

40. A. N. Caruso, C. N. Borca, D. Ristoiu, J. P. Nozieres and P. A. Dowben: Surf. Sci. 525, L109 (2003); D. Ristoiu, J.P. Nozières, C.N. Borca, T. Komesu, H.K. Jeong, and P.A. Dowben: Europhys. Lett. 49, 624 (2000); D. Ristoiu, J.P. Nozières, C.N. Borca, B. Borca, and P.A. Dowben: Appl. Phys. Lett. 76, 2349 (2000); T. Komesu, C.N. Borca, H.-K. Jeong, P.A. Dowben, D. Ristoiu, J.P. Nozières, Sh. Stadler, and Y.U. Idzerda: Phys. Lett. A 273, 245 (2000)

41. I. Galanakis, S. Ostanin, M. Alouani, H. Dreyssé, and J.M. Wills: Phys. Rev. B 61, 4093 (2000)

42. E. Kulatov and I.I. Mazin: J. Phys.: Condens. Matter 2, 343 (1990); S.V. Halilov and E.T. Kulatov: J. Phys.: Condens. Matter 3, 6363 (1991); X. Wang, 
V.P. Antropov, and B.N. Harmon: IEEE Trans. Magn. 30, 4458 (1994). S.J. Youn and B.I. Min: Phys. Rev. B 51, 10436 (1995); V.N. Antonov, P.M. Oppeneer, A.N. Yaresko, A.Ya. Perlov, and T. Kraft: Phys. Rev. B 56, 13012 (1997)

43. P. Larson, S.D. Mahanti, and M.G. Kanatzidis: Phys. Rev. B 62, 12574 (2000)

44. D. Orgassa, H. Fujiwara, T.C. Schulthess, and W.H. Butler: Phys. Rev. B 60, 13237 (1999)

45. I. Galanakis: J. Phys.:Condens. Matter: 14, 6329 (2002)

46. G.A. Wijs and R.A. de Groot: Phys. Rev. B 64, R020402 (2001)

47. A. Debernardi, M. Peressi, and A. Baldereschi: Mat. Sci. Eng. C-Bio S 23, $743(2003)$

48. S.J. Jenkins and D.A. King: Surf. Sci. 494, L793 (2001)

49. S.J. Jenkins and D.A. King: Surf. Sci. 501, L185 (2002)

50. P.J. Webster: J. Phys. Chem. Solids 32, 1221 (1971); K. R.A. Ziebeck and P.J. Webster: J. Phys. Chem. Solids 35, 1 (1974)

51. J.C. Suits: Phys. Rev. B 14, 4131 (1976)

52. J. Kübler, A.R. Williams, and C.B. Sommers: Phys. Rev. B 28, 1745 (1983)

53. S. Ishida, S. Akazawa, Y. Kubo, and J. Ishida: J. Phys. F: Met. Phys. 12, 1111 (1982); S. Ishida, S. Fujii, S. Kashiwagi, and S. Asano: J. Phys. Soc. Jpn. 64, $2152(1995)$

54. S. Fujii, S. Sugimura, S. Ishida, and S. Asano: J. Phys.: Condens. Matter 2, $8583(1990)$

55. S. Fujii, S. Asano, and S. Ishida: J. Phys. Soc. Jpn. 64, 185 (1995)

56. P.J. Brown, K.U. Neumann, P.J. Webster, and K.R.A. Ziebeck: J. Phys.: Condens. Matter 12, 1827 (2000)

57. F. Y. Yang, C. H. Shang, C. L. Chien, T. Ambrose, J. J. Krebs, G. A. Prinz, V. I. Nikitenko, V. S. Gornakov, A. J. Shapiro, and R. D. Shull: Phys. Rev. B 65, 174410 (2002); T. Ambrose, J.J. Krebs, and G.A. Prinz: Appl. Phys. Lett. 76, 3280 (2000); T. Ambrose, J.J. Krebs, and G.A. Prinz: J. Appl. Phys. 87, 5463 (2000); T. Ambrose, J.J. Krebs, and G.A. Prinz: J. Appl. Phys. 89, 7522 (2001)

58. M.P. Raphael, B. Ravel, M.A. Willard, S.F. Cheng, B.N. Das, R.M. Stroud, K.M. Bussmann, J.H. Claassen, and V.G. Harris: Appl. Phys. Lett. 70, 4396 (2001); M.P. Raphael, B. Ravel, Q. Huang, M.A. Willard, S.F. Cheng, B.N. Das, R.M. Stroud, K.M. Bussmann, J.H. Claassen, and V.G. Harris: Phys. Rev. B 66, 104429 (2002); B. Ravel, M.P. Raphael, V.G. Harris, and Q. Huang: Phys. Rev. B 65, 184431 (2002)

59. L. Ritchie, G. Xiao, Y. Ji, T.Y. Chen, C.L. Chien, M. Chang, C. Chen, Z. Liu, G.Wu, and X.X. Zhang: Phys. Rev. B 68, 104430 (2003); Y.J. Chen, D. Basiaga, J.R. O'Brien, and D. Heiman: Appl. Phys. Lett. 84, 4301 (2004)

60. S. Ishida, T. Masaki, S. Fujii, and S. Asano: Physica B 245, 1 (1998)

61. U. Geiersbach, A. Bergmann, and K. Westerholt: J. Magn. Magn. Mater. 240, 546 (2002); U. Geiersbach, A. Bergmann, and K. Westerholt: Thin Solid Films 425, 225 (2003)

62. K. Westerholt, U. Geirsbach, and A. Bergmann: J. Magn. Magn. Mater. 257, 239 (2003)

63. S. Picozzi, A. Continenza, and A.J. Freeman: J. Appl. Phys. 94, 4723 (2003); S. Picozzi, A. Continenza, and A.J. Freeman: J. Phys. Chem. Solids 64, 1697 (2003) 
64. S. Kämmerer, A. Thomas, A. Hütten, and G. Reiss: Appl. Phys. Lett. 85, 79 (2004); J. Schmalhorst, S. Km̈merer, M. Sacher, G. Reiss, A. Hütten, and A. Scholl: Phys. Rev. B 70, 024426 (2004)

65. K. Inomata, S. Okamura, R. Goto, and N. Tezuka: Jpn. J. Appl. Phys. 42, L419 (2003); K. Inomata, N. Tezuka, S. Okamura, H. Kurebayashi, and H. Hirohata: J. Appl. Phys. 95, 7234 (2004)

66. S.H. Vosko, L. Wilk, and N. Nusair: Can. J. Phys. 58, 1200 (1980)

67. P, Hohenberg and W. Kohn: Phys. Rev. 136, B864 (1964); W. Kohn and L.J. Sham: Phys. Rev. 140, A1133 (1965)

68. R. Zeller, P.H. Dederichs, B. Újfalussy, L. Szunyogh, and P. Weinberger: Phys. Rev. B 52, 8807 (1995)

69. N. Papanikolaou, R. Zeller, and P.H. Dederichs: J. Phys.: Condens. Matter 14, 2799 (2002)

70. M.V. Yablonskikh, V.I. Grebennikov, Yu.M. Yarmoshenko, E.Z. Kurmaev, S.M. Butorin, L.-C. Duda, C. Säthe, T. Kämbre, M. Magnuson, J. Nordgren, S. Plogmann, and M. Neumann: Solid State Commun. 117, 79 (2001); M.V. Yablonskikh, Yu.M. Yarmoshenko, V.I. Grebennikov, E.Z. Kurmaev, S.M. Butorin, L.-C. Duda, J. Nordgren, S. Plogmann, and M. Neumann: Phys. Rev. B 63, 235117 (2001)

71. A. Kimura, S. Suga, T. Shishidou, S. Imada, T. Muro, S.Y. Park, T. Miyahara, T. Kaneko, T. Kanomata: Phys. Rev. B 56, 6021 (1997)

72. R.A. de Groot, A.M. van der Kraan, and K.H.J. Buschow: J. Magn. Magn. Mater. 61, 330 (1986)

73. L. Hedin and S. Lundqvist, In: Solid State Physics, vol 23, ed by F. Seitz, D. Turnbull, and H. Ehrenreich, (Academic Press, New York and London 1969) pp 1-181

74. B. R. K. Nanda and I. Dasgupta: J. Phys.: Condens. Matter 15, 7307 (2003)

75. D. Jung, H.-J. Koo, and M.-H. Whangbo: J. Mol. Struct. (Theochem) 527, $113(2000)$

76. J. Kübler: Physica B 127, 257 (1984)

77. D. Brown, M.D. Crapper, K.H. Bedwell, M.T. Butterfield, S.J. Guilfoyle, A.E.R. Malins, and M. Petty: Phys. Rev. B 57, 1563 (1998)

78. R.A. Dunlap and D.F. Jones: Phys. Rev. B 26, 6013 (1982); S. Plogmann, T. Schlathölter, J. Braun, M. Neumann, Yu.M. Yarmoshenko, M.V. Yablonskikh, E.I. Shreder, E.Z. Kurmaev, A. Wrona, and A. Ślebarski: Phys. Rev. B 60, 6428 (1999)

79. P.G. van Engen, K.H.J. Buschow, and M. Erman: J. Magn. Magn. Mater. 30, $374(1983)$

80. W. Pendl Jr., R.N. Saxena, A.W. Carbonari, J. Mestnik Filho, and J. Schaff: J. Phys.: Condens. Matter 8, 11317 (1996)

81. Ye Feng, J.Y. Rhee, T.A. Wiener, D.W. Lynch, B.E. Hubbard, A.J. Sievers, D.L. Schlagel, T.A. Lograsson, and L.L. Miller: Phys. Rev. B 63, 165109 (2001); C.S. Lue, J.H. Ross Jr., K.D.D. Rathnayaka, D.G. Naugle, S.Y. Wu and W.-H. Li: J. Phys.: Condens. Matter 13, 1585 (2001); Y. Nishino, H. Kato, M. Kato, U. Mizutani: Phys. Rev. B 63, 233303 (2001); A. Matsushita, T. Naka, Y. Takanao, T. Takeuchi, T. Shishido, and Y. Yamada: Phys. Rev. B 65, 075204 (2002)

82. S. Picozzi, A. Continenza, and A. J. Freeman: Phys. Rev. B 66, 094421 (2002) 
83. C. Felser, B. Heitkamp, F. Kronast, D. Schmitz, S. Cramm, H. A. Dürr, H.-J. Elmers, G. H. Fecher, S. Wurmehl, T. Block, D. Valdaitsev, S. A. Nepijko, A. Gloskovskii, G. Jakob, G. Schonhense, and W. Eberhardt: J. Phys.:Condens. Matter 15, 7019 (2003); N. Auth, G. Jakob, T. Block T, and C. Felser: Phys. Rev. B 68, 024403 (2003); H. J. Elmers, G. H. Fecher, D. Valdaitsev, S. A. Nepijko, A. Gloskovskii, G. Jakob, G. Schonhense, S. Wurmehl, T. Block, C. Felser, P.-C. Hsu, W.-L. Tsai, and S. Cramm: Phys. Rev. B 67, 104412 (2003); T. Block, C. Felser, G. Jakob, J. Ensling, B. Muhling, P. Gutlich, and R.J. Cava: J. Sol. St. Chem. 176, 646 (2003)

84. R. Kelekar and B.M. Klemens: J. Appl. Phys. 96, 540 (2004)

85. Y. Miura, K. Nagao, and M. Shirai: Phys. Rev. B 69, 144113 (2004); Y. Miura, M. Shirai, and K. Nagao: J. Appl. Phys. 95, 7225 (2004)

86. S. Picozzi, A. Continenza, and A. J. Freeman: Phys. Rev. B 69, 094423 (2004)

87. C.N. Borca, T. Komesu, H.-K. Jeong, P.A. Dowben, D. Ristoiu, Ch. Hordequin, J.P. Nozières, J. Pierre, Sh. Stadler, and Y.U. Idzerda: Phys. Rev. B 64, 052409 (2001)

88. P.A. Dowben and R. Skomski: J. Appl. Phys. 93, 7948 (2003); P.A. Dowben and R. Skomski: J. Appl. Phys. 95, 7453 (2004)

89. Ph. Mavropoulos, K. Sato, R. Zeller, P. H. Dederichs, V. Popescu, and H. Ebert: Phys. Rev. B 69, 054424 (2004); Ph. Mavropoulos, I. Galanakis, V. Popescu, and P. H. Dederichs: J. Phys.: Condens. Matter in press

90. I. Galanakis: unpublished [Preprint arXiv:cond-mat/0405760]

91. C.N. Borca, T. Komesu, and P.A. Dowben: J. Electron. Spectrocs. 122, 259 (2002)

92. L. Chioncel, M.I. Katsnelson, R.A. de Groot, and A.I. Lichtenstein: Phys. Rev. B 68, 144425 (2003) 